May 2012

\title{
Beta2-adrenoceptor agonists for dysmenorrhoea
}

Zbys Fedorowicz

Mona Nasser

Vanitha A. Jagannath

Jessica H. Beaman

Kiran Ejaz

Aga Khan University

See next page for additional authors

Follow this and additional works at: https://ecommons.aku.edu/pakistan_fhs_mc_emerg_med Part of the Emergency Medicine Commons

\section{Recommended Citation}

Fedorowicz, Z., Nasser, M., Jagannath, V., Beaman, J., Ejaz, K., van Zuuren, E. (2012). Beta2-adrenoceptor agonists for dysmenorrhoea. Cochrane Database of Systematic Reviews, 16(5), 1-36.

Available at: https://ecommons.aku.edu/pakistan_fhs_mc_emerg_med/12 
Authors

Zbys Fedorowicz, Mona Nasser, Vanitha A. Jagannath, Jessica H. Beaman, Kiran Ejaz, and Esther J. van Zuuren 


\section{(F) Cochrane Library}

Cochrane Database of Systematic Reviews

\section{Beta2-adrenoceptor agonists for dysmenorrhoea (Review)}

Fedorowicz Z, Nasser M, Jagannath VA, Beaman JH, Ejaz K, van Zuuren EJ

Fedorowicz Z, Nasser M, Jagannath VA, Beaman JH, Ejaz K, van Zuuren EJ.

Beta2-adrenoceptor agonists for dysmenorrhoea.

Cochrane Database of Systematic Reviews 2012, Issue 5. Art. No.: CD008585.

DOI: 10.1002/14651858.CD008585.pub2.

www.cochranelibrary.com

Beta2-adrenoceptor agonists for dysmenorrhoea (Review) 


\section{TABLE OF CONTENTS}

HEADER

ABSTRACT

PLAIN LANGUAGE SUMMARY

BACKGROUND

OBJECTIVES

METHODS

RESULTS

Figure 1.

Figure 2.

Figure 3.

Figure 4.

DISCUSSION

AUTHORS' CONCLUSIONS

ACKNOWLEDGEMENTS

REFERENCES

CHARACTERISTICS OF STUDIES

DATA AND ANALYSES .

Analysis 1.1. Comparison 1 Beta2-adrenoceptor agonist versus placebo, Outcome 1 Reduction in pain. . . . . . 31

APPENDICES . . . . . . . . . . . . . . . . . . . . . . . . . . . . . . . . . . . . .

HISTORY . . . . . . . . . . . . . . . . . . . . . . . . . . . . . . . . . . . . . . . 35

CONTRIBUTIONS OF AUTHORS . . . . . . . . . . . . . . . . . . . . . . . . . . . . . . . . . . . . . . . .

DECLARATIONS OF INTEREST . . . . . . . . . . . . . . . . . . . . . . . . . . . . . . . . . . 35

DIFFERENCES BETWEEN PROTOCOL AND REVIEW . . . . . . . . . . . . . . . . . . . . . . . . . . . . . . $\quad 36$

INDEX TERMS . . . . . . . . . . . . . . . . . . . . . . . . . . . . . . . . . . . 36 


\title{
[Intervention Review]
}

\section{Beta2-adrenoceptor agonists for dysmenorrhoea}

\author{
Zbys Fedorowicz ${ }^{1}$, Mona Nasser ${ }^{2}$, Vanitha A Jagannath ${ }^{3}$, Jessica H Beaman ${ }^{4}$, Kiran Ejaz ${ }^{5}$, Esther J van Zuuren ${ }^{6}$ \\ ${ }^{1}$ UKCC (Bahrain Branch), College of Medicine, AMA International University of Bahrain, Awali, Bahrain. ${ }^{2}$ Peninsula Dental School, \\ University of Plymouth, Plymouth, UK. ${ }^{3}$ Department of Paediatrics, American Mission Hospital, Manama, Bahrain. ${ }^{4}$ The Unversity of \\ Chicago Pritzker School of Medicine, The University of Chicago, Chicago, Illinois, USA. ${ }^{5}$ Emergency Medicine, Aga Khan University \\ Hospital, Karachi, Pakistan. ${ }^{6}$ Department of Dermatology, Leiden University Medical Center, Leiden, Netherlands
}

Contact address: Zbys Fedorowicz, UKCC (Bahrain Branch), College of Medicine, AMA International University of Bahrain, Box 25438, Awali, Bahrain.zbysfedo@batelco.com.bh, zbysfedorowicz@gmail.com.

Editorial group: Cochrane Gynaecology and Fertility Group.

Publication status and date: New, published in Issue 5, 2012.

Citation: Fedorowicz Z, Nasser M, Jagannath VA, Beaman JH, Ejaz K, van Zuuren EJ. Beta2-adrenoceptor agonists for dysmenorrhoea. Cochrane Database of Systematic Reviews 2012, Issue 5. Art. No.: CD008585. DOI: 10.1002/14651858.CD008585.pub2.

Copyright (C) 2012 The Cochrane Collaboration. Published by John Wiley \& Sons, Ltd.

\begin{abstract}
A B S T R A C T
Background

Dysmenorrhoea is a common gynaecological complaint that can affect as many as $50 \%$ of premenopausal women, $10 \%$ of whom suffer severely enough to be rendered incapacitated for one to three days during each menstrual cycle. Primary dysmenorrhoea is where women suffer from menstrual pain but lack any pathology in their pelvic anatomy. Beta2-adrenoceptor agonists have been used in the treatment of women with primary dysmenorrhoea but their effects are unclear.
\end{abstract}

\section{Objectives}

To determine the effectiveness and safety of beta2-adrenoceptor agonists in the treatment of primary dysmenorrhoea.

\section{Search methods}

We searched the Cochrane Menstrual Disorders and Subfertility Group Specialised Register; CENTRAL (The Cochrane Library 2011, Issue 8); MEDLINE; EMBASE; PsycINFO and the EBM Reviews databases. The last search was on 22 August 2011.

\section{Selection criteria}

Randomised controlled trials comparing beta2-adrenoceptor agonists with placebo or no treatment, each other or any other conventional treatment in women of reproductive age with primary dysmenorrhoea.

\section{Data collection and analysis}

Two review authors independently assessed trial quality and extracted the data.

\section{Main results}

Five trials involving 187 women with an age range of 15 to 40 years were included. Oral isoxsuprine was compared with placebo in two trials; terbutaline oral spray, ritodrine chloride and oral hydroxyphenyl-orciprenalin were compared with placebo in a further three trials. Clinical diversity in the studies in terms of the interventions being evaluated, assessments at different time points and the use of different assessment tools mitigated against pooling of outcome data across studies in order to provide a summary estimate of effect for any of the comparisons. Only one study, with unclear risk of bias, reported pain relief with a combination of isoxsuprine, acetaminophen and caffeine. None of the other studies reported any significant clinical difference in effectiveness between the intervention and placebo. Adverse effects were reported with all of these medications in up to a quarter of the total number of participants. They included nausea, vomiting, dizziness, quivering, tremor and palpitations.

Beta2-adrenoceptor agonists for dysmenorrhoea (Review)

Copyright ( 2012 The Cochrane Collaboration. Published by John Wiley \& Sons, Ltd. 


\section{Authors' conclusions}

The evidence presented in this review was based on a few relatively small-sized studies that were categorised to have unclear to high risk of bias, which does not allow confident decision-making at present about the use of beta2-adrenoceptor agonists for dysmenorrhoea. The benefits as reported in one study should be balanced against the wide array of unacceptable side effects documented with this class of medication. We have emphasised the lack of precision and limitations in the reported data where appropriate.

\section{PLAIN LANGUAGE SUMMARY}

\section{Beta2-adrenoceptor agonists for primary dysmenorrhoea}

As many as $50 \%$ of premenopausal women regularly suffer from menstrual pain. Many of these women may be incapacitated for one to three days during each menstrual cycle. Primary dysmenorrhoea (PD) is where women suffer from menstrual pain but lack any pathology in their pelvic anatomy. A wide range of treatments are available and some of these, such as beta2-adrenoceptor agonists, have been used to treat women with primary dysmenorrhoea but their effects are unclear. Five studies involving 187 females with an age range of 15 to 40 years were included in this review. Oral isoxsuprine was examined in two studies; terbutaline oral spray, ritodrine chloride and oral hydroxyphenyl-orciprenalin were compared with placebo in a further three studies. All of the studies were conducted over 30 years ago and none were of high quality. None of these medications, other than isoxsuprine combined with acetaminophen and caffeine, were reported to have any beneficial effect. Side effects with these medications were reported in up to a quarter of the participants and included nausea, vomiting, dizziness, quivering, tremor and palpitations. At present there is insufficient evidence to allow confident decision-making about the use of beta2-adrenoceptor agonists for dysmenorrhoea.

\section{B A C K G ROU N D}

Dysmenorrhoea refers to painful cramps of uterine origin which occur during menstrual periods. The effects of dysmenorrhoea can be debilitating and it is considered to be one of the leading causes of absenteeism from work and school (Hillen 1999; Klein 1981). Interventions that can provide relief from the distressing symptoms that cyclically affect many women of reproductive age could also have a direct bearing on the socioeconomic impact of this condition.

\section{Description of the condition}

\section{Prevalence and aetiology}

Dysmenorrhoea is a common gynaecological complaint that can affect as many as $50 \%$ of premenopausal women, $10 \%$ of whom suffer severely enough to be rendered incapacitated for one to three days during each menstrual cycle (Banikarim 2000; Dawood 1990).

It can be classified as either primary or secondary.

- Primary dysmenorrhoea (PD) is where women suffer from menstrual pain but lack any pathology in their pelvic anatomy.
- Secondary dysmenorrhoea involves a pathological condition (e.g. ovarian cysts, genital lesions) identifiable in the pelvic anatomy (Proctor 2005).

\section{Pathogenesis}

Primary dysmenorrhoea (PD) often starts during adolescence, at six to 12 months after menarche (onset of menstruation) and as soon as ovulatory cycles are established. The duration of pain is usually 48 to 72 hours in a cycle and is associated with the menstrual flow.

The prevalence of PD is highest in adolescent women. Estimates of prevalence range from 20\% to 90\% (Coco 1999; Strinic 2003), and up to $60 \%$ in an adolescent population (aged 12 to 17 years) (Klein 1981). A study in Sweden reported dysmenorrhoea in $90 \%$ of women younger than 19 years of age and in $67 \%$ of women aged 24 years and above (Svanberg 1981). This wide range in the prevalence is most likely due to under reporting of symptoms because many women self-medicate at home and do not seek medical attention. It may also be attributed to differences in criteria used and the lack of standard methods for assessing the severity of dysmenorrhoea (Jamieson 1996).

Risk factors for dysmenorrhoea include:

- positive family history (mother or other female siblings); 
- young age at menarche;

- long menstrual periods with heavy flow;

- smoking;

- obesity;

- alcohol consumption (Andersch 1982; Harlow 1996;

Parazzini 1994).

Some studies have reported that physical activity and the duration of the menstrual cycle do not correlate with increased menstrual pain (Andersch 1982).

\section{Clinical signs, symptoms}

The cramping pain of dysmenorrhoea, which occurs during menstruation (monthly bleeding), tends to be localised to the lower abdomen but may also radiate to the lower back or thighs (Dawood 2006). Other associated symptoms are nausea, vomiting, diarrhoea, headache, fatigue, nervousness and dizziness. The pain can vary from mild to incapacitating, with $32.9 \%$ reporting levels as mild, $49.7 \%$ as moderate, and $17.4 \%$ of sufferers as severe (Ortiz 2009). Symptoms begin shortly before or at the onset of menses and gradually subside over one to three days.

There is growing evidence that the pathogenesis of PD is attributable to prostaglandin F2-alpha (PGF2-alpha), a potent myometrial (uterine muscle) stimulant and vasoconstrictor (contracting blood vessel smooth muscle). PGF2-alpha is present in the secretory endometrium and is capable of enhancing uterine contractions, leading to pain (Coco 1999). The response to prostaglandin inhibitors in patients with dysmenorrhoea supports the assertion that dysmenorrhoea is prostaglandin mediated; and elevated prostaglandin levels found in the endometrial fluid of dysmenorrhoeic women have also been correlated with pain (Eden 1998). The increased levels of prostaglandin in the endometrium following the drop in progesterone in the late phase of the menstrual cycle result in increased myometrial tone and excessive uterine contraction (Dawood 1990).

\section{Description of the intervention}

The beta2-adrenoceptor agonist group of drugs is commonly used for the relief of bronchospasm in patients with asthma, for moderate attacks of dyspnoea and in chronic obstructive pulmonary disease (COPD). These medications have also been found useful in obstetrics because of their effects on the uterine smooth muscle and in delaying the onset of premature labour. Drugs in this category include salbutamol, bitolterol mesylate, formoterol, isoxsuprine, isoprenalin, levalbuterol, metaproterenol, salmeterol, terbutaline and ritodrine (Souney 1983). Common side effects associated with some of these drugs include tremor, agitation, headache and palpitations.

\section{How the intervention might work}

\section{Treatment options for dysmenorrhoea}

A range of treatment options are available, a number of which are the subject of a Cochrane systematic review (Proctor 2007).

\section{Analgesics and nonsteroidal anti-inflammatory drugs (NSAIDs)}

NSAIDs are well established as first-line therapy for primary dysmenorrhoea (Marjoribanks 2010). They have a direct analgesic effect through inhibition of prostaglandin synthesis and are capable of decreasing the volume of menstrual flow. However, whilst their effectiveness has been considered in several reviews (Weaver 2001; Zhang 1998) it has been reported that more than $20 \%$ of dysmenorrhoeic women fail to respond to NSAID therapy (Dawood 2006).

\section{Oral contraceptives}

Off-label use of oral contraceptive pills (OCP) is a recognised treatment option for dysmenorrhoea (Hendrix 2002), however a Cochrane systematic review (Wong 2009) found insufficient evidence from randomised controlled trials to draw conclusions about the effectiveness of OCP in treating dysmenorrhoea.

\section{Other medications and modalities of treatment}

These include thiamine, vitamin E, omega- 3 fatty acids, magnesium, acupuncture, acupressure, various herbal medicines, transdermal nitroglycerin, calcium-channel blockers (Andersson 1978), beta-adrenergic agonists (kerlund 1976), anti-leukotrienes and the use of transcutaneous electrical nerve stimulation (TENS). Use of topical, continuous, low-level heat has also been shown to be beneficial for some women (Akin 2004). Laparoscopic presacral neurectomy (PSN) or uterosacral nerve ablation (LUNA) (Latthe 2007) have been used to treat refractory cases of dysmenorrhoea and have provided a measure of relief for some women for as long as 12 months after treatment.

It has been suggested that beta2-adrenoceptor agonists work through a process of increasing blood flow to uterine muscle and in reducing myometrial activity (kerlund 1976). Binding to the beta2-adrenergic receptors causes a rise in the intracellular concentration of the second messenger cyclic adenosine monophosphate (cAMP), which mediates some of the intracellular events resulting in relaxation of uterine muscle and alleviation of the pain or cramps of dysmenorrhoea (Andersson 1982; Revuelta 1999). 


\section{Why it is important to do this review}

Beta2-adrenoceptor agonists have been used in the treatment of women with primary dysmenorrhoea but their effects are unclear. This systematic review sought to assess the clinical effectiveness and safety of this group of medicines for primary dysmenorrhoea.

\section{O B J E C T I V E S}

To determine the effectiveness and safety of beta2-adrenoceptor agonists in the treatment of primary dysmenorrhoea.

\section{METHODS}

\section{Criteria for considering studies for this review}

\section{Types of studies}

Randomised controlled trials (RCTs).

\section{Types of participants}

\section{Inclusion criteria}

Women of reproductive age ( 15 to 49 years) with:

- primary dysmenorrhoea (i.e. no identifiable pelvic pathology as indicated by pelvic examination, ultrasound scans or laparoscopy) and self-reported pain during the majority of the menstrual cycles or for three consecutive menstrual cycles; or moderate to severe primary dysmenorrhoea (pain that does not respond well to analgesics, affects daily activities or has a high baseline score on a validated pain scale).

\section{Exclusion criteria}

Participants diagnosed with secondary dysmenorrhoea (for example associated with fibroids, endometriosis); dysmenorrhoea resulting from the use of an intrauterine device (IUD); or with mild or infrequent dysmenorrhoea.

\section{Types of interventions}

Beta2-adrenoceptor agonists (any dose, frequency, duration and route of administration) in the treatment group versus placebo or no treatment; or against each other or any other conventional treatment. Examples of beta2-adrenoceptor agonists are described under 'Description of the intervention'. We also included any of these interventions if they were used as an add-on or secondary to conventional therapy.

Concomitant medications or alternative treatments such as diet, exercise and other modalities were permitted provided they were equally available to both treatment groups.

\section{Types of outcome measures}

\section{Primary outcomes}

1. Proportion of women reporting improvement in pain or discomfort, or both (severity measured using any internationally recognised and validated visual analogue scale (VAS)).

2. Adverse effects including tremor, agitation, headache, tachycardia, arrhythmias, palpitations, hypokalaemia or any clinically diagnosed toxicity; and any reported unacceptable adverse events associated with any of the interventions. These were to be categorised as mild (not requiring intervention), moderate (requiring treatment) and severe (life threatening or requiring hospitalisation).

\section{Secondary outcomes}

1. Proportion of women reporting absences from work or school and the duration (hours and days) of scholastic or work absenteeism.

2. Quality of life (including patient or parental satisfaction) as assessed by a validated questionnaire.

3. Requirements for additional medication or modalities of treatment.

4. Relief of symptoms other than 'cramp' pain: proportion of women with relief of symptoms.

5. Time to resolution of all symptoms.

All outcomes were evaluated based on:

- immediate relief (in less than 1 day), where data at 3, 6, 12, and 24 hrs were analysed separately;

- late relief (over $24 \mathrm{hrs}$ ) reported over three consecutive cycles, according to the study, where end of study data were to be combined.

\section{Search methods for identification of studies}

\section{Electronic searches}


For the identification of studies to be included or considered for this review, detailed search strategies were developed for each of the databases to be searched.

The MEDLINE and EMBASE search strategies were combined with phases 1 and 2 of the Cochrane sensitive search strategy for RCTs as published in Appendix 5b.2 of the Cochrane Handbook for Systematic Reviews of Interventions 5.1.0 (updated March 2011) (Higgins 2011).

We searched the following databases for relevant trials:

- Menstrual Disorders and Subfertility Group Specialised

Register of controlled trials (to 22 August 2011) (see Appendix

$1)$;

- Cochrane Central Register of Controlled Trials

(CENTRAL) (The Cochrane Library 2011, Issue 8) (see

Appendix 2);

- MEDLINE (via Ovid) (1966 to 22 August 2011) (see

Appendix 3);

- EMBASE (via Ovid) (1980 to 22 August 2011) (see

Appendix 4);

- PsycINFO (from inception to 22 August 2011) (see

Appendix 5);

- EBM Reviews on 22 August 2011, available at http:// www.libraries.iub.edu/ (see Appendix 6).

The last search was conducted on 22 August 2011.

\section{Searching other resources}

\section{Correspondence}

The reference lists of the included studies were also examined but in view of the age of the studies we were unable to contact any of the investigators to ask for details of additional published and unpublished trials.

\section{Language}

There were no language restrictions on included studies and we arranged to translate any that were not in the English language.

\section{Data collection and analysis}

\section{Selection of studies}

Two of us (ZF and VJ) independently assessed the abstracts of studies identified from the searches. We obtained full copies of all relevant and potentially relevant studies and those appearing to meet the inclusion criteria and for which there were insufficient data in the title and abstract to make a clear decision. The full text papers were assessed independently by the two review authors and any disagreement on the eligibility of included studies was resolved through discussion and consensus or, if necessary, through a third author (KE). We excluded those records that did not meet the inclusion criteria and we noted the reasons for their exclusion in the 'Characteristics of excluded studies' table.

\section{Data extraction and management}

We entered study details into the 'Characteristics of included studies' table using RevMan 5 (RevMan 2011) and collected outcome data using a form designed for this purpose.

Data were extracted independently and in duplicate by two review authors ( $\mathrm{ZF}$ and $\mathrm{VJ}$ ) and only entered into RevMan 5 if there was consensus; any disagreements were resolved by consulting with a third review author $(\mathrm{MN})$.

The following details were extracted.

1. Trial methods: (a) method of allocation; (b) masking of participants, trialists, and outcome assessors; (c) exclusion of participants after randomisation, proportion of and reasons for losses at follow-up.

2. Participants: (a) country of origin and study setting; (b) sample size; (c) age; (d) menstrual history; (e) pregnancy history; (f) inclusion and exclusion criteria; (g) education and occupation history.

3. Intervention: (a) type; (b) concentration, dose and frequency, route of administration; (c) duration of intervention and follow-up.

4. Control: (a) type; (b) duration of intervention and followup.

5. Outcomes: (a) primary and secondary outcomes as specified in the Types of outcome measures' section of this review, including any reported adverse effects.

If stated, the sources of funding of the included studies were recorded.

\section{Assessment of risk of bias in included studies}

Two review authors (ZF, VJ) independently assessed risk of bias using the Cochrane Collaboration's tool for assessing risk of bias as described in Section 8.5 of the Cochrane Handbook for Systematic Reviews of Interventions (Higgins 2011). The gradings were compared and we discussed and resolved any inconsistencies in the assessments.

The following domains were assessed as 'low risk of bias', 'unclear' (uncertain risk of bias) or 'high risk of bias'

1. sequence generation;

2. allocation concealment;

3. blinding of participants, personnel;

4. blinding of outcomes assessment;

5. incomplete outcome data;

6. selective outcome reporting;

7. other bias.

We have reported these assessments for each individual study in the 'Risk of bias in included studies'. 
The overall risk of bias of each of the included studies has also been reported according to the following categories.

- Low risk of bias (plausible bias unlikely to seriously alter the results) if all criteria were met.

- Unclear risk of bias (plausible bias that raises some doubt about the results) if one or more criteria were assessed as unclear.

- High risk of bias (plausible bias that seriously weakens confidence in the results) if one or more criteria were not met.

\section{Measures of treatment effect}

Where sufficient reliable data were available, odds ratios (OR) were calculated. For any dichotomous outcome variables reported in each individual study we would calculate the odds ratio (OR) using a modified intention-to-treat (ITT) analysis, that is if the original investigators did not use ITT analysis we would have considered dropouts to be treatment failures. We would also have calculated the summary ORs and 95\% confidence intervals (CIs) using the Cochrane Collaboration's statistical package (RevMan 2011). Numbers needed to treat (NNT) and their $95 \%$ CIs would have been calculated from the pooled OR and its $95 \% \mathrm{CI}$.

\section{Unit of analysis issues}

In studies where outcomes were repeated measures, data for these outcomes were to be analysed for specific time-points, that is at the end of the intervention and at short, medium and long term follow-up. Cross-over studies were eligible for inclusion because the wash-out period between two cycles was almost a month and there was no likelihood of carry-over effect. As some trials reported cross-over design, the log odds ratios and their corresponding standard errors were calculated and entered into the Cochrane software. Where trials mispresented cross-over data as if from a parallel study, the most conservative results were assumed and entered for analysis.

\section{Dealing with missing data}

We were unable to contact any of the investigators in the included studies and therefore data have been presented as reported using an available case population, according to the recommendations in Chapter 16 of the Cochrane Handbook for Systematic Reviews of Interventions 5.1.0 (Higgins 2011).

\section{Assessment of heterogeneity}

We assessed clinical heterogeneity by examining the characteristics of the studies, the similarity between the types of participants, the interventions and the outcomes, as specified in 'Criteria for considering studies for this review'. As there were an insufficient number of studies comparing similar interventions we did not assess statistical heterogeneity between the studies. Statistical heterogeneity would have been assessed using a $\mathrm{Chi}^{2}$ test and the $\mathrm{I}^{2}$ statistic, where $\mathrm{I}^{2}$ values over $50 \%$ indicate moderate to high heterogeneity and heterogeneity would be considered significant with a P value of less than 0.10 (Higgins 2003).

\section{Assessment of reporting biases}

The low number of studies included in this review did not permit an assessment of publication bias. If we had identified a sufficient number of trials for inclusion in this review (at least 10), we would have assessed publication bias according to the recommendations on testing for funnel plot asymmetry as described in Section 10.4.3.1 of the Cochrane Handbook for Systematic Reviews of Interventions (Higgins 2011). If we had then identified asymmetry we would have tried to assess other possible causes and explored these further in the Discussion section of the review, if appropriate.

\section{Data synthesis}

Three of us (ZF, VJ and EvZ) reported the data, as specified in Chapter 9 of the Cochrane Handbook for Systematic Reviews of Interventions (Higgins 2011). We were unable to enter any data in a meta-analysis, but if a sufficient number of trials were identified the following methods would have been applied. For the synthesis and meta-analysis of any quantitative data, we would have used the fixed-effect model if we established that each study was estimating the same treatment effect, otherwise the random-effects model would have been used.

\section{Subgroup analysis and investigation of heterogeneity}

Lack of data did not permit a subgroup analysis but if further data were available we planned to carry out subgroup analyses based on the following categories: age, years after menarche and gestational status.

\section{Sensitivity analysis}

If a sufficient number of studies had been included, we would have undertaken sensitivity analyses to assess the robustness of our review results by repeating the analysis with the following adjustments: exclusion of studies with unclear or inadequate allocation concealment, blinding of outcomes assessment and completeness of follow-up.

\section{R E S U L T S}

\section{Description of studies}


See: 'Characteristics of included studies' and 'Characteristics of excluded studies'.

\section{Results of the search}

The electronic searches retrieved 144 references to studies. After examination of the titles and abstracts of these references, all of the studies which did not match our inclusion criteria and were clearly ineligible were eliminated from the review. Full text copies of the seven remaining potentially eligible trials were obtained and subjected to further evaluation. One (Gallegos-Torres 1975) had been republished in the Spanish language as Flores-Mercado 1975 and a further study (Akerlund 1976) was excluded as it was not a randomised controlled trial. One of the included studies, which was in the Danish language (Andersen 1977), was translated using Google translate (http://translate.google.com.bh).

For further details see the 'Study flow diagram' (Figure 1). 
Figure I. Study flow diagram.

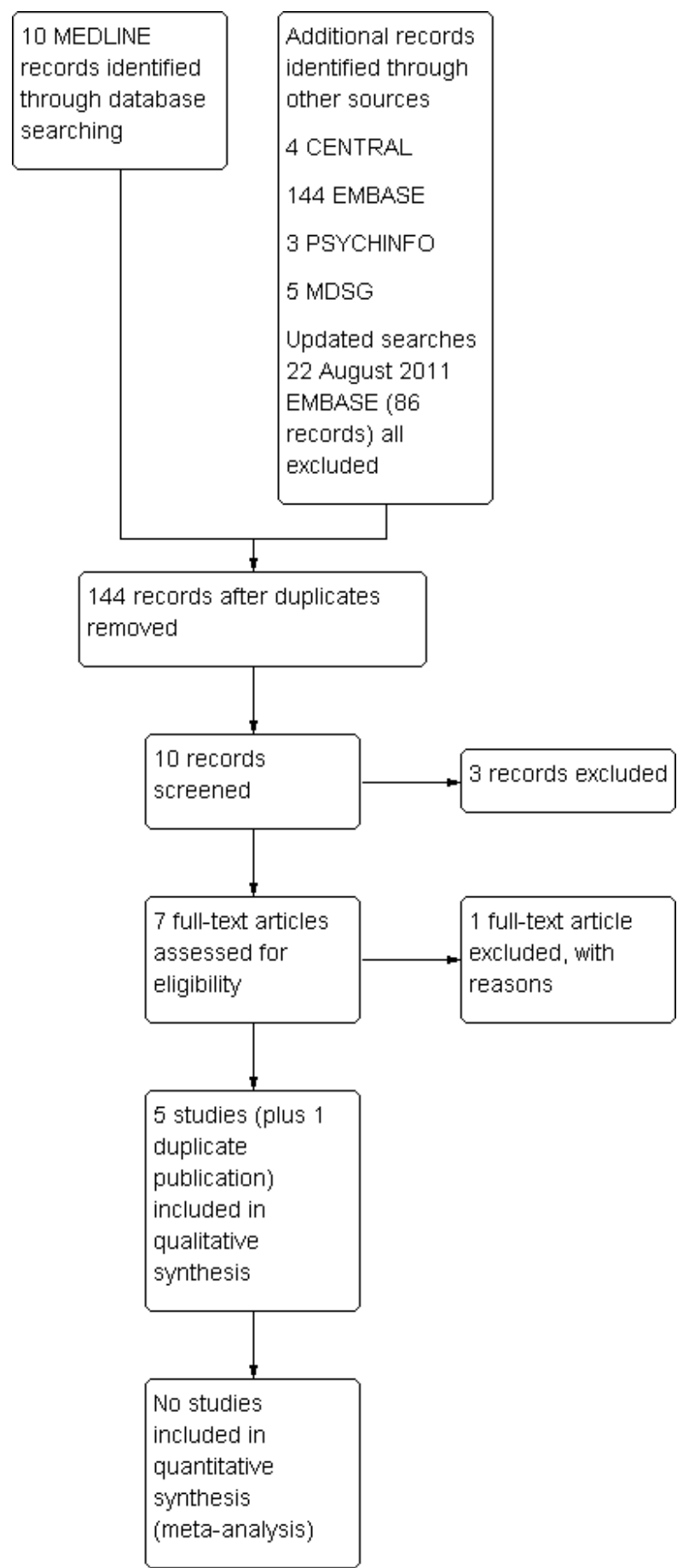




\section{Included studies}

Five studies, all of which had been conducted more than 30 years ago, were included in this review. All five were placebo-controlled. Two (Gallegos-Torres 1975; Hansen 1975) were randomised parallel group trials and the other three (Andersen 1977; Kullander 1981; Nesheim 1976) were randomised but included a cross-over design. One of the studies (Gallegos-Torres 1975) evaluated both the preventive and therapeutic effects of the intervention but on separate subsets of participants. This has been entered into the comparisons as two separate studies.

\section{Characteristics of the trial setting and investigators}

One study (Gallegos-Torres 1975) was conducted in Mexico and the remaining four in Scandinavia. Two studies were conducted in Denmark: Hansen 1975 in a university hospital setting and Andersen 1977 in a county hospital; a general hospital in Sweden was the setting for Kullander 1981; and Nesheim 1976 was conducted in a university hospital in Norway.

\section{Characteristics of the participants}

A total of 187 women with an age range of 15 to 40 years were included. Gravidity and parity of the participants varied between studies; all were nulliparous in Andersen 1977, Hansen 1975 and Nesheim 1976; two-thirds in Kullander 1981; and just over half in Gallegos-Torres 1975 . The duration of a history of primary dysmenorrhoea was not reported in Andersen 1977, Gallegos-Torres 1975, Hansen 1975 or Nesheim 1976 but the participants in Kullander 1981 had severe essential dysmenorrhoea for more than one year. The onset, duration, severity and range of symptoms varied between the participants and across the studies. Participants in one study (Andersen 1977) reported symptoms which occurred over time periods ranging from 12 hours to five days, and half of these were absent from work for at least one day each month. In Gallegos-Torres 1975 the participants had symptoms which persisted from one to 10 days and in Kullander 1981 from one to two days; no details were reported in Hansen 1975 other than that participants spent an unspecified number of days in bed during the course of each cycle. In Nesheim 1976 just over half of the participants had severe dysmenorrhoea, most of whom stayed in bed for one day each menstrual cycle and required analgesic medication for their symptoms.

\section{Characteristics of the interventions}

In two of the studies participants received isoxsuprine orally, over four (Gallegos-Torres 1975) and two (Nesheim 1976) consecutive cycles. A combination of isoxsuprine (10 mg), acetaminophen
(250 mg) and caffeine (30 mg) twice daily, or more as required, was given for one week prior to menstruation in the 'prophylaxis' group and until the appearance of symptoms associated with menstruation in the 'therapy' group (Gallegos-Torres 1975). Participants in Nesheim 1976 received isoxsuprine (20 mg x 3) prior to menstruation and "for as many days as they usually had pain". Oral hydroxyphenyl-orciprenalin $(3 \mathrm{mg})$ followed by a further dose at 30 mins, repeated three to six hourly as required, was provided over four consecutive cycles in Hansen 1975. Six intraoral inhalations of terbutaline ( $1.5 \mathrm{mg}$ ) every $3 \mathrm{~min}$ up to 6 times/day during menstrual pain were used over one cycle and then the participants crossed-over to placebo for the following cycle (Kullander 1981). In Andersen 1977 the participants received ritodrine chloride $(10 \mathrm{mg}$ ) four times daily or placebo to be taken whilst symptoms lasted and were crossed over at the next cycle, for a "total of 60 menstrual cycles". The type and quantity of concomitant analgesic or other permissible medications taken during all of the trials was either unclearly reported or not reported at all.

\section{Characteristics of the outcome measures}

All of the included studies evaluated and reported the primary outcome for this review, that is improvement in pain or discomfort, but these assessments were carried out at different time-points, with a range of assessment tools and reported inconsistently across the studies. Most of these assessments were made using ordinal scales or simple questionnaires, rather than standard internationally recognised generic or disease specific pain scales or visual analogue scales (VAS), none of which appear to have been piloted, tested or validated.

In Gallegos-Torres 1975, although baseline data reported the "nature of pain" and its duration, changes to these were not reported as outcome measures. The "time in which symptoms were relieved" was recorded as a final evaluation at the end of four consecutive cycles but the investigators provided very limited details of these assessments or the tools used. The reliability of any such assessment, based on the possible scaling responses of "excellent, very good, fair, poor" which were available in this instrument, needs to be set in the context of specific concerns about its face and content validity.

Neither the method or assessment tool used nor the timing of any assessment of pain was reported in Hansen 1975 or Kullander 1981. The resolution of pain in Nesheim 1976 was rated simply as "some relief" or "complete relief" but nothing was reported on how or when this evaluation was undertaken. In Andersen 1977, outcomes were reported as "assessments of the effects of treatment" (as translated) and rated on a 4-point ordinal scale ranging from no improvement, significant improvement to completely pain free, but the timing of these assessments was not reported. 
All of the studies noted some of the side effects experienced as a result of the interventions but in most instances these were inadequately reported.

Very few of our secondary outcomes were considered in the included studies. Although it was reported that many of the participants experienced symptoms severe enough to cause absenteeism from work, no data were collected or reported for any of these in any of the studies. No assessments of change or improvement in 'quality of life' as a result of the interventions were carried out. The requirement for additional or concomitant medication was only reported in Kullander 1981, as "some supplementary analgesics", but without any indication of the type, strength, quantity and timing of the use of the medication. The time to resolution of all symptoms was assessed and reported in only one of the included studies (Gallegos-Torres 1975) but in three specific time frames: within the first four hours, 24 hours and between 24 and 48 hours.

\section{Excluded studies}

One study (Akerlund 1976) was excluded (see 'Characteristics of excluded studies').

\section{Risk of bias in included studies}

We assessed each of the included studies for risk of bias and have reported the judgements for each of the individual domains in the 'Assessment of risk of bias in included studies'. These are also presented in the 'Risk of bias' graph in Figure 2 and the 'Risk of bias' summary in Figure 3.

Figure 2. Risk of bias graph: review authors' judgements about each risk of bias item presented as percentages across all included studies.

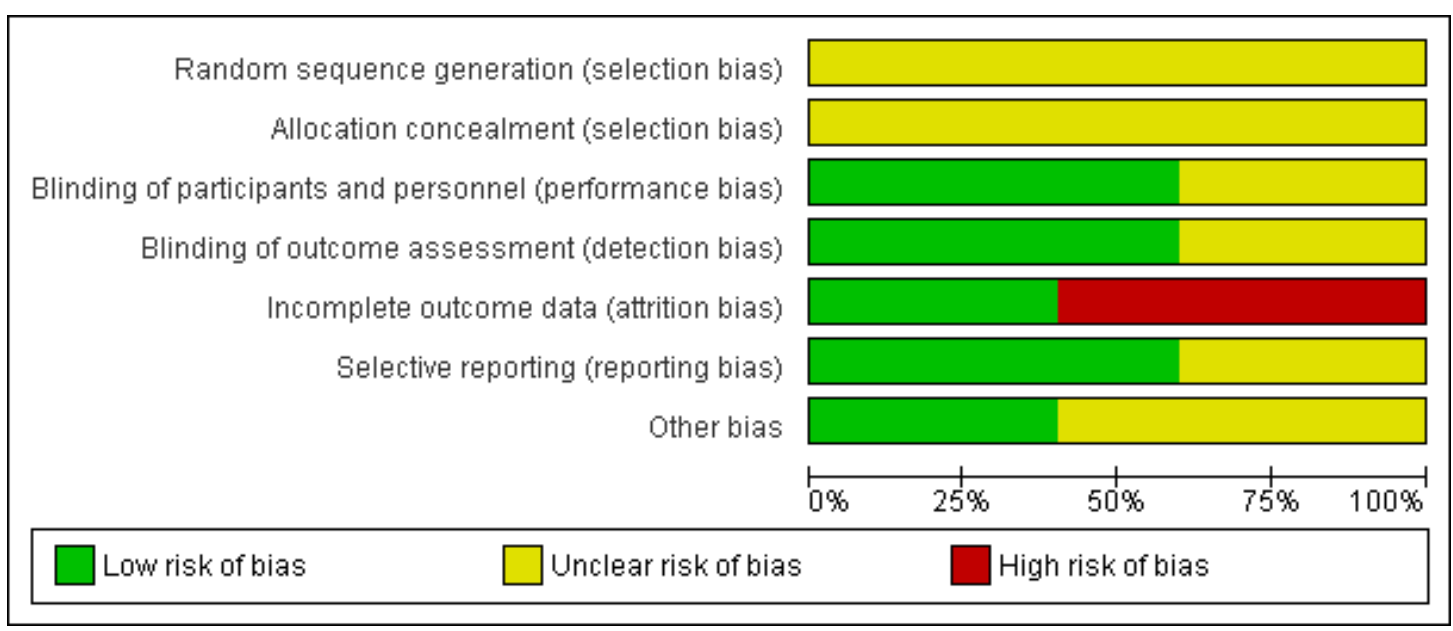


Figure 3. Risk of bias summary: review authors' judgements about each risk of bias item for each included study.

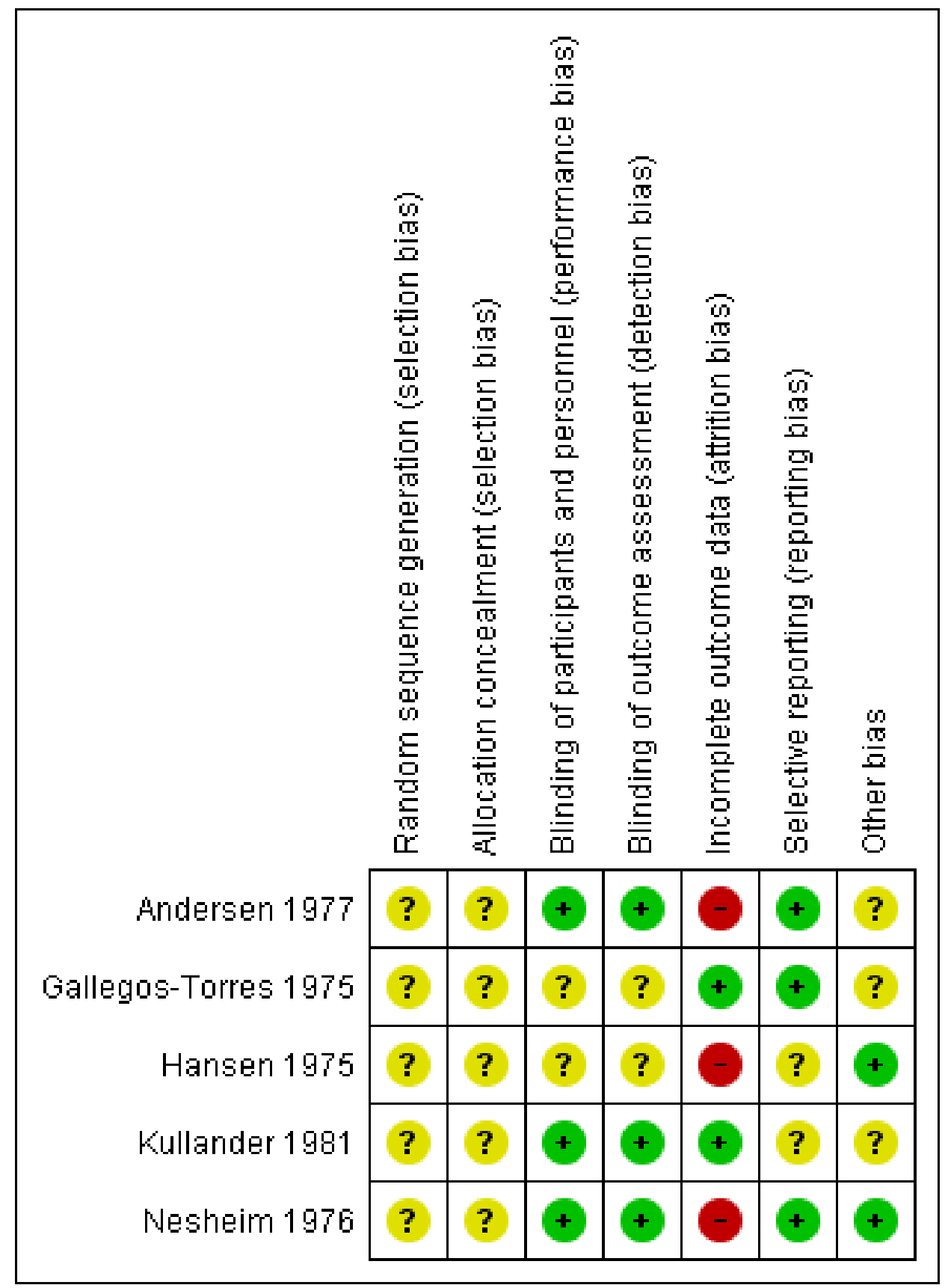

Beta2-adrenoceptor agonists for dysmenorrhoea (Review)

Copyright @ 2012 The Cochrane Collaboration. Published by John Wiley \& Sons, Ltd. 
The overall risk of bias was assessed in each study, and two of the studies (Gallegos-Torres 1975; Kullander 1981) included in this review were categorised as having 'unclear risk of bias' (plausible bias that raises some doubt about the results) because one or more criteria were assessed as 'unclear'. The remaining three studies (Andersen 1977; Hansen 1975; Nesheim 1976) were categorised as at 'high risk of bias' (plausible bias that seriously weakens confidence in the results) because one or more domain received a judgement of 'high risk'.

These assessments were to a certain extent based on the inadequate reporting of the criteria that are a prerequisite in the evaluation of methodological rigour in terms of trial design and conduct. Concealment of the allocation sequence and blinding are key domains in the assessment of risk of bias and most of the studies provided insufficient detail to enable accurate judgements to be made. Protocol deviation, losses to follow-up with incomplete data and subsequent per protocol analyses were other important sources of potential bias in the included studies.

\section{Allocation}

The methods used to generate the allocation sequence and how the sequence was concealed, such that participants and investigators enrolling participants could not foresee the upcoming assignment, are the most important and sensitive indicators that bias has been minimized in a clinical trial (Schulz 1995).

\section{Sequence generation}

The method used to generate the allocation sequence in order to allow an assessment of whether it would produce comparable groups was inadequately reported in all of the studies, which therefore received a judgement of 'unclear' for this domain.

\section{Allocation concealment}

Inadequate reporting in all of the studies did not allow an assessment to be made on whether adequate measures were taken to ensure that investigators were unaware of the upcoming assignment. Therefore, we judged this domain as 'unclear' risk of bias for all of the included studies.

\section{Blinding}

Two of the reports (Gallegos-Torres 1975; Hansen 1975) did not provide sufficient information, other than that the studies were "double blind", to enable a clear assessment of whether adequate and effective measures were used to blind study participants and personnel from knowledge of which intervention a participant received. The three remaining studies (Andersen 1977; Kullander 1981; Nesheim 1976) reported that the interventions were of "identical appearance", which indicated that the intended blinding was probably effective and therefore judgements of 'low risk' of performance bias were given to these studies. All of the outcomes assessments reported in the included studies were carried out by the participants. In three of the studies (Andersen 1977; Kullander 1981; Nesheim 1976) the participants appeared to have been effectively blinded and therefore this domain in these studies was judged as 'low risk' of detection bias, whereas in the two other studies it was assessed as 'unclear' risk of bias.

\section{Incomplete outcome data}

In Gallegos-Torres 1975 all 80 participants contributed outcome data so we judged this domain as 'low risk' of bias. Although in Kullander 1981 one patient "discontinued treatment because of side effect", the data appeared to be otherwise complete for each main outcome and this domain was judged 'low risk' of bias. In three of the studies (Andersen 1977; Hansen 1975; Nesheim 1976) protocol violations or withdrawals and missing outcome data in addition to per protocol analyses represented a 'high risk' of bias for this domain.

\section{Selective reporting}

The study protocol was not available for any of the included studies but in two of the reports (Hansen 1975; Kullander 1981) the outcomes sought were not clearly specified in the methods section, which did not permit a clear judgement of risk of bias to be made for this domain in these studies. In the remaining studies all outcomes specified in the methods section appeared to have been reported fully, with no evidence of selective reporting, and the studies were judged 'low risk' of bias for this domain.

\section{Other potential sources of bias}

Poorly defined study outcome measures and a lack of clarity in their assessment were some of the other potential threats to the validity of the included trials that were not addressed by the other domains in this risk of bias assessment tool. Although the investigators did not indicate any sources of study support or sponsorship, or declare any conflicts of interest, some study medications were supplied by pharmaceutical manufacturers. There was no evidence of any untoward influence on the design, conduct or analysis in any of the included studies.

In Kullander 1981, results from the cross-over study were misreported as if it they had arisen from a parallel group trial. In Gallegos-Torres 1975, although not described as a within-participant comparison, it looks most likely that a similar error occurred. We judged this domain as 'unclear' risk of bias for both studies. 


\section{Effects of interventions}

All of the studies included in this review were categorised as either 'unclear' or 'high' risk of bias and therefore caution is advised in the interpretation of their findings and in the extrapolation of the effects of the interventions to clinical decision-making. Two studies evaluated the effects of orally administered isoxsuprine compared with placebo (Gallegos-Torres 1975; Nesheim 1976). Terbutaline spray as an oral inhalation was compared with placebo in a cross-over study (Kullander 1981); and in the remaining studies, ritodrine chloride (Andersen 1977) and oral hydroxyphenylorciprenalin (Hansen 1975) were compared with placebo.

Clinical diversity in the studies in terms of the interventions being evaluated, assessments at different time-points and the use of different assessment tools mitigated against pooling of outcome data across studies in order to provide a summary estimate of effect for any of the comparisons. We have presented outcomes in a narrative fashion and emphasised the limitations and lack of precision in the reported data where appropriate.

\section{(I) Comparison of oral ritodrine chloride with placebo}

One study evaluated this comparison (Andersen 1977) and reported two primary outcomes but none of the secondary outcomes.

\section{Primary outcomes}

\section{Proportion of women reporting improvement in pain or discomfort}

Athough the investigators reported that there was no statistically significant difference in improvement in 'effects' between the two groups (author stated $\mathrm{P}$ value $=0.89$ ), the data for the two periods in this cross-over study were inadequately reported and analysed. Albeit the potential impact of any cross-over or period effect is unclear, these were not addressed in the data analysis in this study. Furthermore, the lack of data in this study did not permit its reanalysis or the calculation of an effect estimate, and therefore these have not been reported.

\section{Adverse effects}

Nine participants in the ritodrine group reported palpitations and tremors, and two in the placebo group experienced mild palpitations.

\section{Secondary outcomes}

No data were reported.

\section{(2) Comparison of oral isoxsuprine combined with acetaminophen and caffeine with placebo}

One study (Gallegos-Torres 1975) evaluated this comparison and reported the two primary outcomes and one of the secondary outcomes.

\section{Primary outcomes}

\section{Proportion of women reporting improvement in pain or discomfort}

This outcome was rated as excellent, very good, fair or poor based on time to complete resolution, rather than change in severity of symptoms, expressed as the measure of degree of improvement. In the prophylaxis and therapy studies, up to $95 \%$ and $92.5 \%$ of the participants reported improvement with isoxsuprine whereas $40 \%$ and $42.5 \%$ reported improvement with the placebo, respectively. For estimated effect size, the odds of having an 'excellent' or 'very good' outcome were compared assuming the most conservative estimate from a paired comparison that was compatible with the presented tables for each of the prophylaxis and therapy trials. This corresponded to assuming that none of the two and three participants, respectively, without a good outcome on active therapy also failed to have a good outcome on placebo (OR 11.94, 95\% CI 2.01 to 71.06 for prophylactic comparison; OR 5.64, 95\% CI 1.31 to 24.06 for therapeutic comparison; Analysis 1.1, Figure 4). 
Figure 4. Forest plot of comparison: I Beta2-adrenoceptor agonist versus placebo, outcome: I.I Reduction in pain.

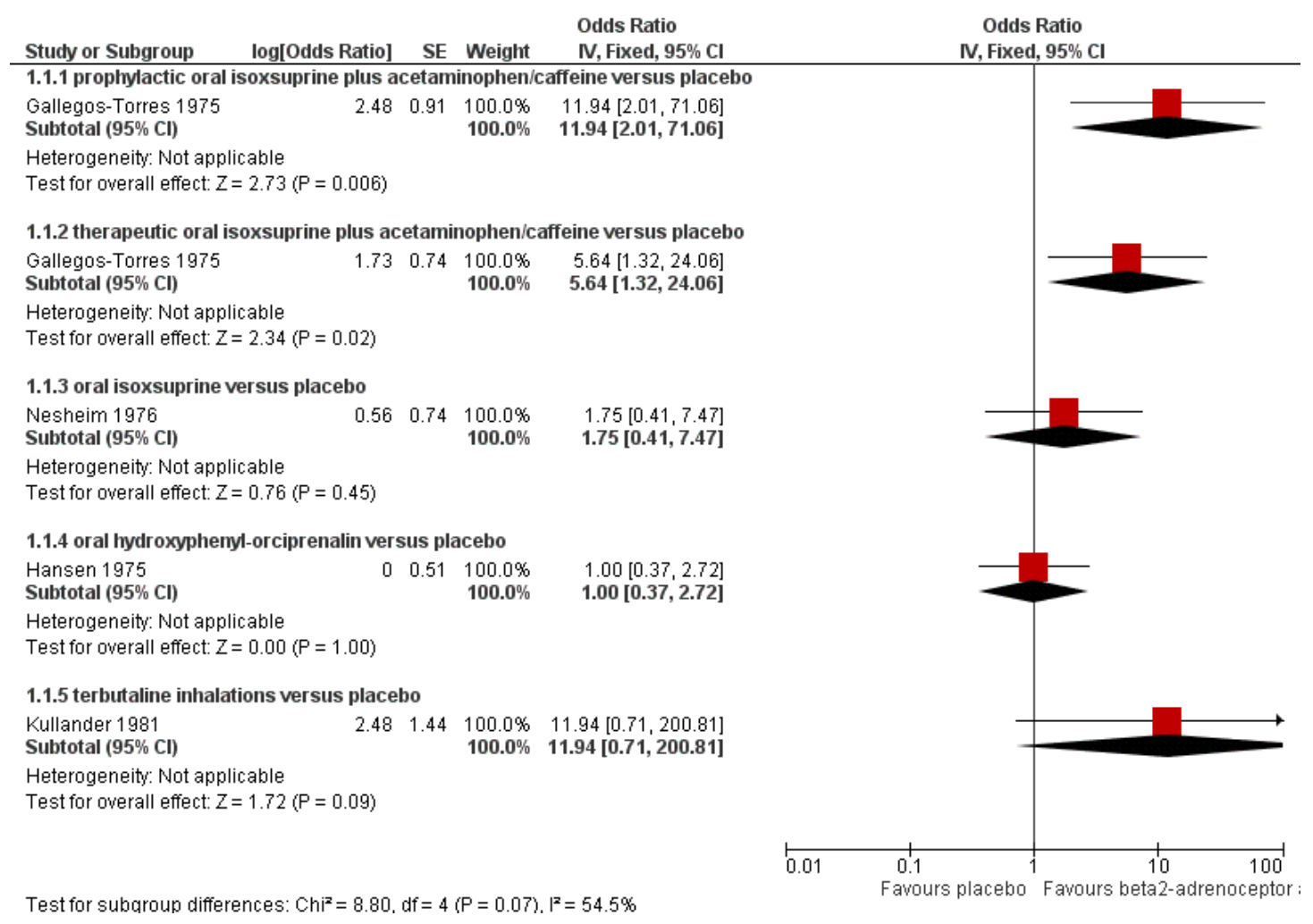

\section{Adverse effects}

Nausea and vomiting were the only side effects with the intervention, which occurred in the prophylaxis study in $15 \%$ of participants and in the therapy study in $10 \%$ of the participants.

\section{Secondary outcomes}

\section{Time to resolution of symptoms}

Where symptom resolution was rated as 'excellent' in the prophylaxis group, symptoms were relieved within the first four hours in $72.5 \%$ of those who received oral isoxsuprine compared with $22.5 \%$ receiving placebo. In the therapy group this was $70 \%$ of those treated with isoxsuprine; and none in the placebo group.
One study (Nesheim 1976) evaluated this comparison and reported the two primary outcomes but none of the secondary outcomes for this review.

Primary outcomes

\section{Proportion of women reporting improvement in pain or discomfort}

Only 8/26 participants had "some relief" of symptoms with isoxsuprine compared to 5/26 with placebo (OR $1.75,95 \%$ CI 0.41 to 7.47; Analysis 1.1). The data for the two periods in this crossover study were inadequately reported and analysed. Albeit the potential impact of any cross-over or period effects is unclear, these were not addressed in the data analysis in this study.

\section{(3) Comparison of oral isoxsuprine with placebo}

Adverse effects

Beta2-adrenoceptor agonists for dysmenorrhoea (Review) 
Almost 20\% (5/26) of the participants experienced some side effects whilst on isoxsuprine. The reported side effects in the isoxsuprine group were dizziness, quivering, nausea and headache.

\section{Secondary outcomes}

No data were reported.

\section{(4) Comparison of oral hydroxyphenyl-orciprenalin with placebo}

Only one study evaluated this comparison (Hansen 1975), and two primary outcomes but none of the secondary outcomes were reported.

\section{Primary outcomes}

\section{Proportion of women reporting improvement in pain or discomfort}

The investigators reported that 10 of the 47 participants $(\mathrm{N}=$ 70) who completed the trial in the hydroxyphenyl-orciprenalin group had a "good effect", three of whom were completely relieved of pain. A similar number (10) in the placebo group achieved a similar "effect". However the large number of losses (32\%) and corresponding missing data were not taken into consideration in the analysis and it was unclear how many participants were randomised in each group (OR 1.00, 95\% CI 0.37 to 2.72; Analysis 1.1).

\section{Adverse effects}

Almost half (25) of the participants experienced palpitations whilst receiving hydroxyphenyl-orciprenalin.

\section{Secondary outcomes}

No data were reported.

\section{(5) Comparison of terbutaline oral inhalations with placebo}

One study evaluated this comparison (Kullander 1981), and two primary outcomes and one secondary outcome were reported.

\section{Primary outcomes}

\section{Proportion of women reporting improvement in pain or discomfort}

The trialists reported that terbutaline provided an "alleviating effect" for 12 out of 14 participants, and 1 out of 14 participants in the placebo group. Odds of success were compared using the most conservative estimate from a paired comparison that was compatible with the presented table. This corresponded to assuming that the participant who responded to placebo failed to respond to terbutaline (OR 11.94, 95\% CI 0.71 to 200.81; Analysis 1.1).

\section{Adverse effects}

Slight tremor and palpitations were experienced by three participants and in one case a flush during the terbutaline period.

\section{Secondary outcomes}

\section{Requirements for additional medication}

Over half (8) of the participants required supplementary medication for complete relief of symptoms during the terbutaline period and almost all (12/14) during the placebo period.

\section{DISCUSSION}

\section{Summary of main results}

Our comprehensive search for randomised controlled trials which examined the effects of the interventions specified in the protocol for this review identified a number of trials. In one of these the investigators endorsed the effectiveness of a combination of isoxsuprine with acetaminophen and caffeine but provided very limited and poor quality outcome data to substantiate these claims (Gallegos-Torres 1975). We were unable to obtain adequate data from most of the trials and we summarise only the results from our analysis of several of the outcomes from these trials. The results in the other study investigating isoxsuprine (Nesheim 1976) failed to show any benefit; as was the case in the remaining studies, which evaluated ritodrine (Andersen 1977), hydroxyphenyl-orciprenalin (Hansen 1975) and terbutaline (Kullander 1981). Adverse effects with all of these medications were reported in up to a quarter of the total number of participants and included nausea, vomiting, dizziness, quivering, tremor and palpitations. 


\section{Overall completeness and applicability of evidence}

This review included five trials, most of which were conducted over 30 years ago and whilst they did not provide any reliable evidence to support the effectiveness of these interventions they did report some of their undesirable side effects.

\section{Quality of the evidence}

\section{Limitations in study design and implementation}

Although the study design in the included studies appeared to have been at best adequate, our study-level assessments of the risk of bias for a number of the domains in several of these studies revealed some of the limitations in their implementation. These have been reported in the 'Risk of bias in included studies' section of this review.

\section{Inconsistency of results}

One of the included studies (Gallegos-Torres 1975), which was also republished as Flores-Mercado 1975, reported consistent and positive effects for isoxsuprine that were more positive than the conclusions reached in Nesheim 1976. Other than the duplicate publication of the Gallegos-Torres 1975 study, and based on its over positive results, one might question the apparent lack of follow-up studies by the trialists to further confirm their results.

\section{Indirectness of evidence}

The evidence for effectiveness is somewhat limited and is focused on a comparison of the effects of beta2-adrenoceptor agonists versus placebo. We do not have any direct evidence on the comparative effectiveness of any of the beta2-adrenoceptor agonists and we did not attempt to conduct any indirect comparison (network meta analysis) to extrapolate the potential comparative effectiveness of different beta2-adrenoceptor agonists against each other. The participants in the included studies were in general a representative sample, as defined in the inclusion criteria, and therefore we did not have any significant concerns about the directness of participants identified in the review. All of the included studies matched the eligibility criteria for this review. Four studies were conducted in hospital settings in European countries (Denmark, Sweden and Norway) and only one of them was conducted in a developing country (Mexico), in which the setting was not specified. This may be an issue for consideration by guideline groups that develop guidelines for populations in a primary care setting in developing countries. However, it should be kept in mind that downgrading the evidence for differences in populations is not recommended except if there are important reasons indicating that these differences might result in substantial changes in the magnitude of effect of the intervention (Guyatt 2011).

\section{Imprecision of results}

The small number and poor quality of the studies that were included in this review did not provide any data which could appropriately be pooled and therefore any substantive assessment of the degree of precision of effect was not feasible. We have exercised caution when extracting and reporting data from the primary research studies and have indicated, where appropriate, any uncertainty with the reliability of the data and any conclusions which can be made from them.

\section{Publication bias}

Although it would be reasonable to assume that the comprehensive electronic searches employed in this review will have identified all existing randomised controlled trials, and thereby helped to limit bias in the conduct of this review, the absence of any other published trials over the intervening 25 years and the scant contribution of the five included trials to the outcomes specified for this review might be a cause for concern about publication bias. Moreover, even though only a small number of trials were identified and an assessment of publication bias could not be made, one cannot safely discount the possible existence of some unpublished studies with either similar, that is null to negative, results or reporting additional side effects.

\section{Potential biases in the review process}

We made every attempt to limit bias in the review process by ensuring a comprehensive search for potentially eligible studies. The authors' independent assessments of eligibility of studies for inclusion in this review and the extraction of data from the included studies minimized the potential for additional bias beyond that detailed in the 'Risk of bias in included studies' tables. The incompleteness of some of the reports and our inability to obtain clarification of certain trial details or to resolve ambiguities in the reports may have contributed to some bias in their assessment. Where these conditions applied this was explicitly stated in the text of our review. The effects of language bias on the identification and selection of studies is widely recognised and therefore we ensured that any studies that were not in the English language were translated so that they could be assessed for eligibility and risk of bias. 


\section{Agreements and disagreements with other studies or reviews}

We are unaware of any other published systematic reviews or any additional studies assessing the effectiveness of these interventions. We have examined several evidence supported guidelines on the management of dysmenorrhoea, none of which considered beta2adrenoceptor agonists.

\section{AUTHORS' CONCLUSIONS}

\section{Implications for practice}

The evidence presented in this review, which was based on a few relatively small-sized studies categorised as having unclear to high risk of bias, does not allow confident decision-making about the use of beta2-adrenoceptor agonists for dysmenorrhoea. However, although these studies may have reported a measure of pain relief and somewhat limited clinical improvement in some of the participants these should be balanced against the wide array of unacceptable side effects that have been documented with this class of medications. This may be a reason why no further studies have been carried out and most probably why beta2-adrenoceptor agonists are, in general, no longer considered the first-line treatment for dysmenorrhoea. Until, and if, further evidence becomes available, clinicians should continue to base their decisions on currently recommended therapeutic regimens of oral contraceptives and NSAIDS, and other mainstays of treatment which include reassurance and education, some of which have been covered in other Cochrane reviews.

\section{Implications for research}

Although we make no specific recommendations for future research, proof of concept in vitro studies which explore the potential of a transvaginal mode of delivery of terbutaline to limit systemic side effects may provide additional treatment options which should be considered in future research on this clinical topic (Bulletti 2001). If conducted, these trials should be well-designed and adequately delivered with subsequent reporting including high-quality descriptions of all aspects of methodology. Rigorous reporting needs to conform to the Consolidated Standards of Reporting Trials (CONSORT) statement (http://www.consortstatement.org/), which will enable appraisal and interpretation of results and accurate judgements to be made about the risk of bias and the overall quality of the evidence. Although it is uncertain whether reported quality mirrors actual study conduct, it is noteworthy that studies with unclear methodology have been shown to produce biased estimates of treatment effects (Schulz 1995). Adherence to guidelines, such as the CONSORT statement, would help ensure complete reporting.

\section{ACKNOWLEDGEMENTS}

We would like to acknowledge the support we have received from the Cochrane Menstrual Disorders and Subfertility Group in developing the protocol and conducting this review. Andy Vail abstracted data and calculated odds ratios for the effect estimates but took no other part in the review.

\section{R E F E R E N C E S}

\section{References to studies included in this review}

\section{Andersen 1977 \{published data only\}}

Andersen AN. Essential dysmenorrhoea. Severe essential dysmenorrhoea treated with the beta-mimetic agent ritodrine [Danish] [Svær primær dysmenoré behandlet med den selektive beta-adrenergic stimulator ritodrinklorid]. Ugeskrift for Laeger 1977;139(23):1366-8.

Gallegos-Torres 1975 \{published data only\}

Flores-Mercado F, Gallegos-Torres J. Isoxsuprine in primary dysmenorrhea. Its efficiency in premenstrual tension [Spanish] [La Isoxsuprina en dismenorrea primaria]. Ginecologia y Obstetricia de Mexico 1975;38(228):279-87.

* Gallegos-Torres J, Flores-Mercado F. Isoxsuprine in primary dysmenorrhoea. Its effectiveness in premenstrual tension. Journal of International Medical Research 1975;3 (3):194-201.
Hansen 1975 \{published data only\} Hansen MK, Secher NJ. Beta-receptor stimulation in essential dysmenorrhea. American Journal of Obstetrics and Gynecology 1975;121(4):566-7.

Kullander 1981 \{published data only\} Kullander S, Svanberg L. Terbutaline inhalation for alleviation of severe pain in essential dysmenorrhea. Acta Obstetricia et Gynecologica Scandinavica 1981;60(4):425-7.

Nesheim 1976 \{published data only\}

Nesheim BI, Walloe L. The use of isoxsuprine in essential dysmenorrhea. A controlled clinical study. Acta Obstetricia et Gynecologica Scandinavica 1976;55(4):315-6.

\section{References to studies excluded from this review}

Akerlund 1976 \{published data only\}

Akerlund M, Andersson KE, Ingemarsson I. Effects of terbutaline on myometrial activity, uterine blood flow, and lower abdominal pain in women with primary 
dysmenorrhoea. British Journal of Obstetrics and Gynaecology 1976;83(9):673-8.

\section{Additional references}

\section{Akin 2004}

Akin M, Price W, Rodriguez G Jr, Erasala G, Hurley G, Smith RP. Continuous, low-level, topical heat wrap therapy as compared to acetaminophen for primary dysmenorrhea. Journal of Reproductive Medicine 2004;49(9):739-45. [PUBMED: 15493566]

\section{Andersch 1982}

Andersch B, Milsom I. An epidemiologic study of young women with dysmenorrhea. American Journal of Obstetrics and Gynecology 1982;144(6):655-60. [PUBMED: 7137249]

\section{Andersson 1978}

Andersson KE, Ulmsten U. Effects of nifedipine on myometrial activity and lower abdominal pain in women with primary dysmenorrhoea. British Journal of Obstetrics and Gynaecology 1978;85:142-8.

\section{Andersson 1982}

Andersson KE. Pharmacological inhibition of uterine activity. Acta Obstetricia et Gynecologica Scandinavica 1982; 108 Suppl:17-23. [PUBMED: 6126985]

\section{Banikarim 2000}

Banikarim C, Chacko MR, Kelder SH. Prevalence and impact of dysmenorrhea on Hispanic female adolescents. Archives of Pediatrics and Adolescent Medicine 2000;154: 1226-9.

\section{Bulletti 2001}

Bulletti C, de Ziegler D, de Moustier B, Polli V, Bolelli G, Franceschetti F, et al. Uterine contractility: vaginal administration of the beta-adrenergic agonist, terbutaline. Evidence of direct vagina-to-uterus transport. Annals of the New York Academy of Sciences 2001;943:163-71. [PUBMED: 11594537]

\section{Coco 1999}

Coco AS. Primary dysmenorrhea. American Family Physician 1999;60:489-96.

\section{Dawood 1990}

Dawood MY. Dysmenorrhea. Clinical Obstetrics and Gynecology 1990;33(1):168-78.

\section{Dawood 2006}

Dawood MY. Primary dysmenorrhea: advances in pathogenesis and management. Obstetrics and Gynecology 2006;108(2):428-41.

\section{Eden 1998}

Eden JA. Dysmenorrhea and premenstrual syndrome. Essentials of Obstetrics and Gynecology. 3rd Edition. Philadelphia: WB Saunders, 1998.

\section{Guyatt 2011}

Guyatt GH, Oxman AD, Kunz R, Woodcock J, Brozek J, et al. The GRADE Working Group. GRADE guidelines: 8. Rating the quality of evidence-indirectness. Journal of Clinical Epidemiology 2011;64(12):1303-10. [DOI: 10.1016/j.jclinepi.2011.04.014; PMID: 21802903

Harlow 1996

Harlow SD, Park M. A longitudinal study of risk factors for the occurrence, duration and severity of menstrual cramps in a cohort of college women. British Journal of Obstetrics and Gynaecology 1996;103(11):1134-42. [PUBMED: 8917003]

\section{Hendrix 2002}

Hendrix SL, Alexander NJ. Primary dysmenorrhea treatment with a desogestrel-containing low-dose oral contraceptive. Contraception 2002;66:393-9.

\section{Higgins 2003}

Higgins JP, Thompson SG, Deeks JJ, Altman DG. Measuring inconsistency in meta-analyses. BMJ 2003;327 (7414):557-60

\section{Higgins 2011}

Higgin JPT, Green S, editors. Cochrane Handbook for Systematic Reviews of Interventions Version 5.1.0 [updated March 2011]. The Cochrane Collaboration, 2011. Available from www.cochrane-handbook.org. Available from www.cochrane-handbook.org, 2011.

\section{Hillen 1999}

Hillen TI, Grbavac SL, Johnston PJ, Straton JA, Keogh JM. Primary dysmenorrhoea in young Western Australian women: prevalence, impact and knowledge of treatment. Journal of Adolescent Health 1999;25(1):40-5.

\section{Jamieson 1996}

Jamieson DJ, Steege JF. The prevalence of dysmenorrhea, dyspareunia, pelvic pain, and irritable bowel syndrome in primary care practices. Obstetrics and Gynecology 1996;87 (1):55-8.

\section{Klein 1981}

Klein JR, Litt IF. Epidemiology of adolescent dysmenorrhea. Pediatrics 1981;68(5):661-4.

\section{Latthe 2007}

Latthe PM, Proctor ML, Farquhar CM, Johnson N, Khan KS. Surgical interruption of pelvic nerve pathways in dysmenorrhea: a systematic review of effectiveness. Acta Obstetricia et Gynecologica Scandinavica 2007;86(1):4-15. [PUBMED: 17230282]

\section{Marjoribanks 2010}

Marjoribanks J, Proctor ML, Farquhar C, Derks RS. Nonsteroidal anti-inflammatory drugs for dysmenorrhoea. Cochrane Database of Systematic Reviews 2010, Issue 1. [DOI: 10.1002/14651858.CD001751.pub2

\section{Ortiz 2009}

Ortiz MI, Rangel-Flores E, Carrillo-Alarcon LC, VerasGodoy HA. Prevalence and impact of primary dysmenorrhea among Mexican high school students. International Journal of Gynaecology and Obstetrics 2009;107(3):240-3. [PUBMED: 19716130]

\section{Parazzini 1994}

Parazzini F, Tozzi L, Mezzopane R, Luchini L, Marchini M, Fedele L. Cigarette smoking, alcohol consumption, and 
risk of primary dysmenorrhea. Epidemiology (Cambridge, Massachusetts) 1994;5(4):469-72. [PUBMED: 7918820]

\section{Proctor 2005}

Proctor M, Latthe P, Farquhar CM, Khan KS, Johnson N. Surgical interruption of pelvic nerve pathways for primary and secondary dysmenorrhoea. Cochrane Database of Systematic Reviews 2005, Issue 4. [DOI: 10.1002/ 14651858.CD001896.pub2

Proctor 2007

Proctor ML, Farquhar CM. Dysmenorrhoea. Clinical Evidence (online) 2007;1:pii: 0813.

RevMan 2011 [Computer program]

The Nordic Cochrane Centre, The Cochrane Collaboration. Review Manager (RevMan). Version 5.1. Copenhagen: The Nordic Cochrane Centre, The Cochrane Collaboration, 2011.

\section{Revuelta 1999}

Revuelta MP, Hidalgo A, Cantabrana B. Involvement of cAMP and beta-adrenoceptors in the relaxing effect elicited by flavonoids on rat uterine smooth muscle. Journal of Autonomic Pharmacology 1999;19(6):353-8. [PUBMED: 10961741]

\section{Schulz 1995}

Schulz KF, Chalmers I, Hayes RJ, Altman DG. Empirical evidence of bias. Dimensions of methodological quality associated with estimates of treatment effects in controlled trials. JAMA 1995;273(5):408-12.

\section{Souney 1983}

Souney PF, Kaul AF, Osathanondh R. Pharmacotherapy of preterm labor. Clinical Pharmacy 1983;2(1):29-44. [PUBMED: 6136361]

\section{Strinic 2003}

Strinic T, Bukovic D, Pavelic L, Fajdic J, Herman I, Stipic I. Anthropological and clinical characteristics in adolescent women with dysmenorrhea adolescent women with dysmenorrhea Anthropological and clinical characteristics in adolescent women with dysmenorrhea. Collegium Antropologicum 2003;27:707-11.

\section{Svanberg 1981}

Svanberg L, Ulmsten U. The incidence of primary dysmenorrhea in teenagers. Archives of Gynecology 1981; 230(3):173-7.

\section{Weaver 2001}

Weaver AL. Rofecoxib: clinical pharmacology and clinical experience. Clinical Therapeutics 2001;23:1323-38.

\section{Wong 2009}

Wong CL, Farquhar C, Roberts H, Proctor M. Oral contraceptive pill as treatment for primary dysmenorrhoea. Cochrane Database of Systematic Reviews 2009, Issue 2. [DOI: 10.1002/14651858.CD002120.pub2

\section{Zhang 1998}

Zhang WY, Li Wan Po A. Efficacy of minor analgesics in primary dysmenorrhoea: a systematic review. British Journal of Obstetrics and Gynaecology 1998;105:780-9.

\section{kerlund 1976}

kerlund M, Andersson KE, Ingemarsson I. Effects of terbutaline on myometrial activity, uterine blood flow, and lower abdominal pain in women with primary dysmenorrhoea. British Journal of Obstetrics and Gynaecology 1976;83(9):673-8.

* Indicates the major publication for the study 


\section{CHARACTERISTICS OF STUDIES}

\section{Characteristics of included studies [ordered by study ID]}

\section{Andersen 1977}

Methods

Randomised, double-blind placebo-controlled cross-over trial in Denmark. County hospital setting, date unspecified, over a total number of 60 cycles

Participants

RANDOMISED: 26, age 15-36 yrs

INCLUSION CRITERIA:

- primary dysmenorrhoea with symptoms requiring regular medication and resulting in absenteeism from work

- regular cycle

- nulliparous

- no abnormal gynaecological problems i.e. endometriosis, fibromyoma EXCLUSION CRITERIA:

- using intrauterine contraception or other OCP

- on hormone therapy

DROP OUTS/WITHDRAWALS: 6; pregnancy(1), side effects of the intervention(1), lost to follow-up (4)

Interventions

Interventions delivered over 60 cycles in 20 participants but not reported how many cycles/participant

INTERVENTION:

- ritodrine chloride $(10 \mathrm{mg})$ qid taken whilst symptoms persist COMPARISON

- placebo

Crossed-over at the next cycle

Outcomes

Timing and frequency of outcome assessment unreported PRIMARY OUTCOMES:

"a special form" was used to assess the "impact" and "effects of treatment" and side effects Rating scale: no improvement/improvement/completely pain free

Adverse effects in intervention and control groups were reported

Absenteeism, quality of life and requirement for additional medication unreported

Notes

No sponsorship/support or declaration of any conflicts of interest reported. Authors have not accounted for/explained the cross-over or period effects in the analysis

Risk of bias

\begin{tabular}{|c|c|c|}
\hline Bias & Authors' judgement & Support for judgement \\
\hline $\begin{array}{l}\text { Random sequence generation (selection } \\
\text { bias) }\end{array}$ & Unclear risk & $\begin{array}{l}\text { Quote: "randomised" Pg } 1366 . \\
\text { Comment: Insufficient detail reported } \\
\text { about the method used to generate the al- } \\
\text { location sequence }\end{array}$ \\
\hline
\end{tabular}


Allocation concealment (selection bias) Unclear risk

Blinding of participants and personnel Low risk (performance bias)

All outcomes

Blinding of outcome assessment (detection Low risk bias)

All outcomes

Incomplete outcome data (attrition bias) High risk All outcomes
The method used to conceal the allocation sequence, that is to determine whether intervention allocations could have been foreseen in advance of, or during enrolment, was not reported.

Comment: Insufficient information to permit a clear judgement

Quote: "double-blind" and "tablets identical” Pg 1366.

Comment: Probably done.

Quote: "double-blind" and "tablets identical” Pg 1366.

Outcomes assessment:

- Participant-assessed

Review authors judge that the intended blinding of participants and key personnel was probably effective and unlikely to introduce bias into the outcome assessment.

Comment: Withdrawals/dropouts 6/26 (32\%) but number per group, reasons reported only (2/26). Paucity of data and lack of clarity in its analysis i.e. whether the participants were analysed according to the intention-to-treat principle High risk of bias.
Although the study protocol was unavailable all outcomes specified in the methods section appear to have been reported and there was no evidence of selective reporting

Other bias Unclear risk
The outcomes assessments were poorly defined and relevant data inadequately reported, unclear if these may represent concerns about bias not addressed in the other domains in this tool Unclear risk of bias.

\section{Gallegos-Torres 1975}

Methods
Randomised, double-blind placebo-controlled trial in Mexico. Date and setting unreported. Duration of study four consecutive menstrual cycles 
Participants

Participants

RANDOMISED: 80 , age $16-40$ yrs

2 groups of 40: "prophylactic administration and therapeutic administration" Within groups divided into 20 placebo, 20 drug combination INCLUSION CRITERIA:

- primary or essential dysmenorrhoea with or without pre-menstrual tension and no pelvic disease EXCLUSION CRITERIA:

- secondary dysmenorrhoea (organo-genic/acquired/extrinsic)

- anovulatory cycles

DROP OUTS/WITHDRAWALS: None reported
Two groups: Prophylactic (A) before onset of symptoms; Therapeutic (B) after the onset of symptoms

Group A1 active intervention (20). Group A2 placebo (20)

Group B1 active intervention (20). Group B2 placebo (20)

INTERVENTION:

- oral isoxsuprine $(10 \mathrm{mg})$, acetaminophen $(250 \mathrm{mg})$, caffeine $(30 \mathrm{mg})$ in combination

COMPARISON

- placebo

Prophylactic Group (A) 12 hourly or more often if symptoms present, starting one week before the onset of periods

Therapeutic Group (B) 12 hourly after onset of symptoms in each cycle

Intervention over four consecutive menstrual cycles

Outcomes

Final evaluation at the end of four consecutive cycles, but very limited information, how and in particular which assessment tools were used

PRIMARY OUTCOMES:

- number of participants rated improvement/change: excellent (symptoms completely relieved); very good (symptoms failed to disappear entirely); fair and poor (no improvement observed). Severity measured as nature and duration of pain

- adverse effects

SECONDARY OUTCOMES:

- time to resolution of all symptoms

Absenteeism, quality of life and requirement for additional medication unreported

Notes

No sponsorship/support or declaration of any conflicts of interest reported

\section{Risk of bias}

Bias

Random sequence generation (selection Unclear risk bias)
Support for judgement

Quote: "patients were distributed at random into two groups...” Pg 195.

Comment: Insufficient detail reported about the method used to generate the allocation sequence 

sequence, that is to determine whether intervention allocations could have been foreseen in advance of, or during enrolment, was not reported.

Comment: Insufficient information to permit a clear judgement

Blinding of participants and personnel Unclear risk (performance bias)

All outcomes
Quote: “double-blind method...” Pg 194. Comment: The measures used to blind study participants and personnel from knowledge of which intervention a participant received were not clearly reported Participants were outcome assessors. Review authors judge it is unclear if the outcome is likely to be influenced by the lack of blinding

Insufficient information to permit a clear judgement. Unclear risk of bias

Blinding of outcome assessment (detection Unclear risk bias)

All outcomes

Low risk

Incomplete outcome data (attrition bias) Low risk All outcomes

Selective reporting (reporting bias)

Unclear risk

Comment: The measures used to blind the outcome assessors from knowledge of which intervention a participant received were not clearly reported

Outcomes assessment:

- Participant-assessed

Lack of blinding is likely to exert an influence on outcome measurement, but insufficient detail reported to permit a clear judgement.

Unclear risk of bias.

No evidence of missing or incomplete data due to attrition or exclusions for the outcomes reported in the study.

Comment: Low risk of bias.

Although the study protocol was unavailable all outcomes specified in the methods section appear to have been reported and there was no evidence of selective reporting

Other bias
There are clearly 80 participants with 40 in each of the prophylaxis and therapy studies. However, each study reports results as if from a parallel trial with 40 in each treatment group. Although in the methods section there is no mention of crossover design it seems most likely that each 


\section{Methods}

Participants
Randomised double-blind placebo-controlled trial in Odensee University Hospital, Denmark. Date unreported. Duration of study four consecutive menstrual cycles

Student nurses 17-28 yrs nulliparous

RANDOMISED: $\mathrm{N}=70$, unclear how many to each group INCLUSION CRITERIA:

- lower abdominal, cramp like pains, using analgesics, occasional bed rest at time of menstruation

EXCLUSION CRITERIA:

- none reported

DROP OUTS/WITHDRAWALS:

- $23 / 70(32 \%)$ due to protocol violation i.e. use of "additional analgesics or started hormonal contraception during study" Pg 566

Interventions

INTERVENTION:

- oral hydroxyphenyl-orciprenalin $(3 \mathrm{mg})$ followed by a further dose at $30 \mathrm{mins}$, repeated 3-6 hourly prn

CONTROL:

- placebo

Four consecutive menstrual cycles this study at 'high risk of bias

\section{Risk of bias}

$\begin{array}{lll}\text { Bias Authors' judgement Support for judgement } & \text { Suld }\end{array}$

Random sequence generation (selection Unclear risk bias)
Quote: "The distribution of the drug and placebo was randomized”. Pg 567.

Comment: Insufficient detail reported about the method used to generate the al- 
Hansen 1975 (Continued)

location sequence

Allocation concealment (selection bias) Unclear risk

The method used to conceal the allocation sequence, that is to determine whether intervention allocations could have been foreseen in advance of, or during enrolment, was not reported.

Comment: Insufficient information to permit a clear judgement

Blinding of participants and personnel Unclear risk (performance bias)

All outcomes

Quote: “double-blind”. Pg 566.

Comment: The measures used to blind study participants and personnel from knowledge of which intervention a participant received were not clearly reported

Participants were outcome assessors. Review authors judge it is unclear if the outcome is likely to be influenced by the lack of blinding

Insufficient information to permit a clear judgement.

Unclear risk of bias.

Blinding of outcome assessment (detection Unclear risk bias)

All outcomes
Comment: The measures used to blind the outcome assessors from knowledge of which intervention a participant received were not clearly reported

Outcomes assessment:

- Participant-assessed.

Review authors judge it is unclear if the outcome assessment is likely to be influenced by the lack of blinding. Unclear risk of bias

Incomplete outcome data (attrition bias) High risk All outcomes
Quote: “excluded were those who, despite the instructions given either used additional and not clearly defined analgesics or started hormonal contraception during study". Pg 566

Comment: Withdrawals/dropouts and protocol violations $(32 \%)$ but number per group and the reasons unreported. Paucity of data and lack of clarity in its analysis i.e. whether the participants were analysed according to the intention to treat principle High risk of bias.

The protocol was unavailable and the reported outcomes were not pre-specified in the methods section of the study.

Beta2-adrenoceptor agonists for dysmenorrhoea (Review) 
Kullander 1981

\begin{tabular}{|c|c|c|}
\hline Methods & \multicolumn{2}{|c|}{$\begin{array}{l}\text { Randomised, double-blind placebo-controlled cross-over trial in Sweden. Date unre- } \\
\text { ported, in General Hospital setting. Duration of study two consecutive menstrual cycles }\end{array}$} \\
\hline Participants & \multicolumn{2}{|c|}{$\begin{array}{l}\text { RANDOMISED: N=14, aged 15-39 yrs (mean 27), } 10 \text { nulliparous, } 4 \text { parous } \\
\text { INCLUSION CRITERIA: } \\
\text { • "severe essential dysmenorrhea for more than one year". Pg } 425 \\
\text { EXCLUSION CRITERIA: none reported } \\
\text { DROP OUTS/WITHDRAWALS: none reported }\end{array}$} \\
\hline Interventions & \multicolumn{2}{|c|}{$\begin{array}{l}\text { Active intervention one menstrual period and crossed-over to placebo for next period } \\
\text { INTERVENTION: } \\
\text { - terbutaline ( } 1.5 \mathrm{mg} \text { ), six intra-oral inhalations every } 3 \text { min up to } 6 \text { times/day } \\
\text { during menstrual pain prn } \\
\text { CONTROL: } \\
\text { - placebo }\end{array}$} \\
\hline Outcomes & \multicolumn{2}{|c|}{$\begin{array}{l}\text { PRIMARY OUTCOMES: } \\
\text { therapeutic effect was assessed and graded as none/weak/moderate/good very good at } \\
\text { the end of each menstrual cycle } \\
\text { - pain relief } \\
\text { - adverse effects were recorded } \\
\text { SECONDARY OUTCOMES: } \\
\text { - reported as supplementary analgesia, but no details of type and dosage provided } \\
\text { Absenteeism, quality of life and requirement for additional medication unreported }\end{array}$} \\
\hline Notes & \multicolumn{2}{|c|}{ No sponsorship/support or declaration of any conflicts of interest reported } \\
\hline \multicolumn{3}{|l|}{ Risk of bias } \\
\hline Bias & Authors' judgement & Support for judgement \\
\hline $\begin{array}{l}\text { Random sequence generation (selection } \\
\text { bias) }\end{array}$ & Unclear risk & $\begin{array}{l}\text { Quote: "were given in random order....". } \\
\text { Pg } 425 . \\
\text { Method used to generate the allocation se- } \\
\text { quence. Unclear if all participants received } \\
\text { active intervention in the first period and } \\
\text { "placebo sprays .....during the next period" }\end{array}$ \\
\hline
\end{tabular}


Kullander 1981 (Continued)

Allocation concealment (selection bias) Unclear risk

Blinding of participants and personnel Low risk (performance bias)

All outcomes

Blinding of outcome assessment (detection Low risk bias)

All outcomes

Incomplete outcome data (attrition bias) Low risk

All outcomes
The method used to conceal the allocation sequence, that is to determine whether intervention allocations could have been foreseen in advance of, or during enrolment, was not reported.

Comment: Insufficient information to permit a clear judgement

Quote: "sprays of identical appearance...." Pg 425 .

Comment: Probably done.

Quote: "sprays of identical appearance...." Pg 425.

Outcomes assessment:

- Participant-assessed.

Review authors judge that the intended blinding of participants and key personnel was probably effective and unlikely to introduce bias into the outcome assessment.

Quote: “one patient discontinued treatment because of side effect" Pg 427.

No other losses to attrition or exclusions were reported, the data appear to have been otherwise complete for each main outcome.

Comment: Low risk of bias.

Selective reporting (reporting bias) Unclear risk

The study protocol was unavailable and the outcomes sought were not clearly specified in the methods which did not permit a clear judgement to be made.

Comment: Unclear risk of bias.

Other bias

Unclear risk
Although this was a cross-over design the presented analysis is as if it were a parallel design with twice as many participants. Comment: Unclear risk of bias.

Nesheim 1976

Methods

Participants
Randomised, double-blind placebo-controlled cross-over trial in Oslo Norway. Date and setting unspecified. Duration of study two consecutive menstrual cycles

RANDOMISED: 30 (29 nulliparous, 1 primiparous), severe (18), moderate (12) dysmenorrhoea. Age and other demographic details unreported INCLUSION CRITERIA: 
Nesheim 1976 (Continued)

\[ \text { not reported } \]
EXCLUSION CRITERIA:
$\bullet$ not reported
DROPOUTS/WITHDRAWALS: 4 due to side effects; placebo (1), and (3) intervention
group and reasons not reported

Notes

No sponsorship/support or declaration of any conflicts of interest reported other than intervention and placebo supplied by Ferrosan/Norfarma A/S

\section{Risk of bias}

Bias

Random sequence generation (selection Unclear risk bias)

\section{Support for judgement}

Quote: "The type of tablets taken during the first cycle was randomized". Pg 315. Comment: Insufficient detail reported about the method used to generate the allocation sequence

Allocation concealment (selection bias) Unclear risk

tinding of participants and personnel Low risk (performance bias)

All outcomes
The method used to conceal the allocation sequence, that is to determine whether intervention allocations could have been foreseen in advance of, or during enrolment, was not reported.

Comment: Insufficient information to permit judgement.

Quote: “isoxsuprine $20 \mathrm{mg} \mathrm{x} 3$ or placebo tablets identical in appearance" and "double-blind”. Pg 315.

Comment: Probably done. 
Blinding of outcome assessment (detection Low risk bias)

All outcomes
Quote: “isoxsuprine $20 \mathrm{mg} \mathrm{x} 3$ or placebo tablets identical in appearance" and "double-blind". Pg 315

Outcomes assessment:

- Participant-assessed.

Review authors judge that the intended blinding of participants and key personnel was probably effective and unlikely to introduce bias into the outcome assessment.

Incomplete outcome data (attrition bias) High risk All outcomes

Quote: "Twenty six of the participants completed the trial. One of those who did not complete did so because of side effects of the first course of the tablets (which was placebo), for the other three the reasons were not related to tablets”. Pg 315

Comment: Although losses to follow up were reported and small although unbalanced, the numbers in each group and reasons, unclear.

Paucity of data and lack of clarity in its analysis i.e. whether the participants were analysed according to the intention to treat principle

High risk of bias.
Although the study protocol was unavailable all outcomes specified in the methods section appear to have been reported and there was no evidence of selective reporting

Although medication and placebo were supplied by the manufacturer there was no evidence of any influence over the design, conduct or data analysis in the trial

\section{Characteristics of excluded studies [ordered by study ID]}

\begin{tabular}{l|l}
\hline Study & Reason for exclusion \\
\hline Akerlund 1976 & Non-RCT \\
\hline
\end{tabular}

RCT $=$ Randomised controlled trial

$\mathrm{OCP}=$ Oral contraceptive pill

prn = pro re nata (when necessary)

Beta2-adrenoceptor agonists for dysmenorrhoea (Review)

Copyright @ 2012 The Cochrane Collaboration. Published by John Wiley \& Sons, Ltd. 
DATA AND ANALYSES

Comparison 1. Beta2-adrenoceptor agonist versus placebo

\begin{tabular}{|c|c|c|c|c|}
\hline Outcome or subgroup title & $\begin{array}{l}\text { No. of } \\
\text { studies }\end{array}$ & $\begin{array}{c}\text { No. of } \\
\text { participants }\end{array}$ & Statistical method & Effect size \\
\hline 1 Reduction in pain & 4 & & Odds Ratio (Fixed, 95\% CI) & Subtotals only \\
\hline $\begin{array}{l}1.1 \text { prophylactic } \\
\text { oral isoxsuprine plus } \\
\text { acetaminophen/caffeine versus } \\
\text { placebo }\end{array}$ & 1 & & Odds Ratio (Fixed, 95\% CI) & $11.94[2.01,71.06]$ \\
\hline $\begin{array}{l}1.2 \text { therapeutic } \\
\text { oral isoxsuprine plus } \\
\text { acetaminophen/caffeine versus } \\
\text { placebo }\end{array}$ & 1 & & Odds Ratio (Fixed, 95\% CI) & $5.64[1.32,24.06]$ \\
\hline $\begin{array}{l}1.3 \text { oral isoxsuprine versus } \\
\text { placebo }\end{array}$ & 1 & & Odds Ratio (Fixed, 95\% CI) & $1.75[0.41,7.47]$ \\
\hline $\begin{array}{l}1.4 \text { oral hydroxyphenyl- } \\
\text { orciprenalin versus placebo }\end{array}$ & 1 & & Odds Ratio (Fixed, 95\% CI) & $1.0[0.37,2.72]$ \\
\hline $\begin{array}{l}1.5 \text { terbutaline inhalations } \\
\text { versus placebo }\end{array}$ & 1 & & Odds Ratio (Fixed, 95\% CI) & $11.94[0.71,200.81]$ \\
\hline
\end{tabular}


Analysis I.I. Comparison I Beta2-adrenoceptor agonist versus placebo, Outcome I Reduction in pain.

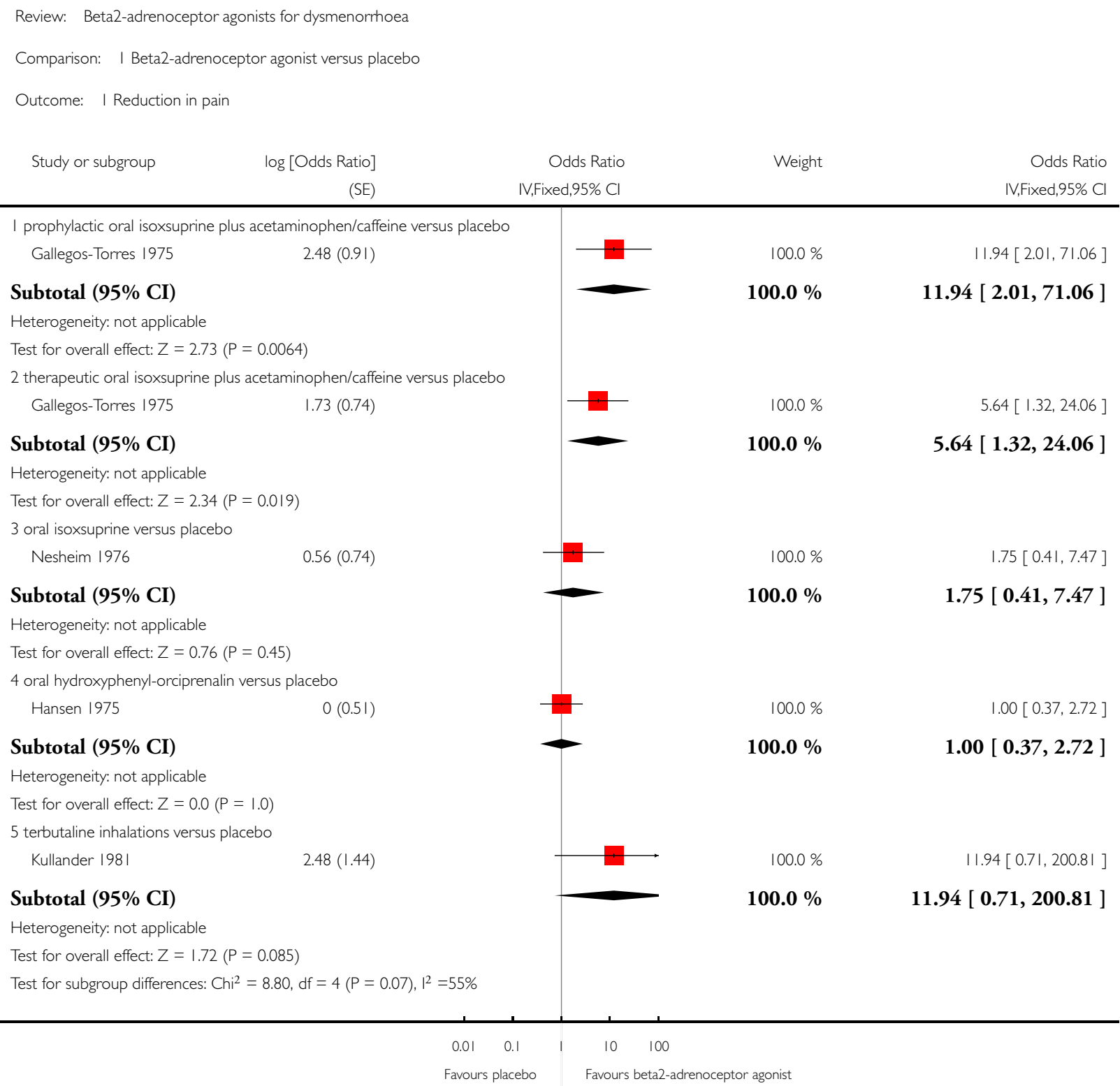




\section{A P P E N D I C E S}

\section{Appendix I. Menstrual Disorders and Subfertility Group (MDSG) Specialised Register}

The MDSG search string is dated from inception of database until present.

Keywords CONTAINS “*Dysmenorrhea” or "Dysmenorrhea-Symptoms" or "dysmenorrhoea” or "cyclical pelvic pain" or "primary dysmenorrhea" or "pelvic pain" or "pain-dysmenorrhea" or "pain-pelvic" or "menstrual distress" or "menstrual pain" or "menstrual cramps" or "cramping" or Title CONTAINS“*Dysmenorrhea” or "Dysmenorrhea-Symptoms" or "dysmenorrhoea” or "cyclical pelvic pain" or "primary dysmenorrhea" or "pelvic pain" or "pain-dysmenorrhea" or "pain-pelvic" or "menstrual distress" or "menstrual pain" or "menstrual cramps" or "cramping"

AND

Keywords CONTAINS "Terbutaline" or "Isoxsuprine" or "beta-adrenoceptor" or Title CONTAINS or "Terbutaline" or "Isoxsuprine" or "beta-adrenoceptor"

\section{Appendix 2. CENTRAL search strategy}

The CENTRAL search string is dated from inception of database until 22 August 2011.

1 Pelvic Pain/

2 Dysmenorrhea/

3 painful menstruat $\$ . t i, a b, s h$.

4 (pelvi\$ adj5 pain).ti,ab,sh.

5 dysmenorrh\$.ti,ab,sh.

6 (cyclic\$ adj1 pain\$).tw.

7 or/1-6

8 exp Albuterol/

9 exp Adrenergic beta-Agonists/

10 salbutamol.tw.

11 Terbutaline.tw.

12 albuterol.tw.

13 levalbuterol.tw.

14 proventil.tw.

15 sultanol.tw.

16 ventolin.tw.

17 xopenex.tw.

18 Adren $\$$ beta.tw.

19 or/8-18

207 and 19

\section{Appendix 3. MEDLINE (via Ovid) search strategy}

The MEDLINE search string is dated from 1950 until 22 August 2011.

1 Pelvic Pain/

2 Dysmenorrhea/

3 painful menstruat $\$ . t i, a b, s h$.

4 (pelvi\$ adj5 pain).ti,ab,sh.

5 dysmenorrh\$.ti,ab,sh.

6 (cyclic\$ adj1 pain\$).tw.

7 or/ $1-6$

8 exp Albuterol/

9 exp Adrenergic beta-Agonists/

10 salbutamol.tw.

11 Terbutaline.tw.

Beta2-adrenoceptor agonists for dysmenorrhoea (Review)

Copyright @ 2012 The Cochrane Collaboration. Published by John Wiley \& Sons, Ltd. 
12 albuterol.tw.

13 levalbuterol.tw.

14 proventil.tw.

15 sultanol.tw.

16 ventolin.tw.

17 xopenex.tw.

18 Adren $\$$ beta.tw.

19 or/8-18

20 randomized controlled trial.pt.

21 controlled clinical trial.pt.

22 randomized.ab.

23 placebo.tw.

24 clinical trials as topic.sh.

25 randomly.ab.

26 trial.ti.

27 (crossover or cross-over or cross over).tw.

28 or/ $20-27$

29 (animals not (humans and animals)).sh.

3028 not 29

317 and 19 and 30

\section{Appendix 4. EMBASE (via Ovid) search strategy}

1 exp dysmenorrhea/ or exp menstruation disorder/ or exp pain/

2 dysmenorrh\$.tw.

3 (pelvi\$ adj5 pain).tw.

4 (painful adj5 menstrua\$).tw.

5 (pain\$ adj5 period\$).tw.

6 (cycl\$ adj2 pain\$).tw.

7 (menstr\$ adj2 cramp\$).tw.

8 or/ $1-7$

9 exp beta adrenergic receptor/

10 exp salbutamol sulfate/ or exp salbutamol/

11 salbutamol.tw.

12 albuterol.tw.

13 Terbutaline.tw.

14 levalbuterol.tw.

15 Isoxsuprine.tw.

16 proventil.tw.

17 sultanol.tw.

18 ventolin.tw.

19 xopenex.tw.

20 Adren\$ beta $\$ . t w$.

21 (beta\$ adj2 agonist\$).tw.

22 or/9-21

238 and 22

24 Clinical Trial/

25 Randomized Controlled Trial/

26 exp randomization/

27 Single Blind Procedure/

28 Double Blind Procedure/

29 Crossover Procedure/

Beta2-adrenoceptor agonists for dysmenorrhoea (Review)

Copyright $(2012$ The Cochrane Collaboration. Published by John Wiley \& Sons, Ltd. 
30 Placebo/

31 Randomi?ed controlled trial\$.tw.

32 Rct.tw.

33 random allocation.tw.

34 randomly allocated.tw.

35 allocated randomly.tw.

36 (allocated adj2 random).tw.

37 Single blind\$.tw.

38 Double blind\$.tw.

39 ((treble or triple) adj blind\$).tw.

40 placebo\$.tw.

41 prospective study/

42 or/ $24-41$

43 case study/

44 case report.tw.

45 abstract report/ or letter/

46 or/ $43-45$

4742 not 46

4823 and 47

$49(201006 \$$ or $201007 \$$ or $201008 \$$ or $201009 \$$ or $201010 \$$ or $201011 \$$ or $201012 \$)$.em.

$502011 \$ . \mathrm{em}$

5149 or 50

5248 and 51

\section{Appendix 5. PsycINFO search strategy}

The PsycINFO search strategy is dated from 1806 until 22 August 2011

1 exp dysmenorrhea/ or exp menstrual disorders/

2 dysmenorrh\$.tw.

3 (pelvi\$ adj5 pain).tw.

4 (painful adj5 menstrua\$).tw.

5 (pain\$ adj5 period\$).tw.

6 (cycl\$ adj2 pain\$).tw.

7 (menstr\$ adj2 cramp\$).tw.

8 or/ $1-7$

9 exp adrenergic drugs/ or exp adrenergic receptors/

10 beta adren $\$$.tw.

11 salbutamol.tw.

12 albuterol.tw.

13 Terbutaline.tw.

14 levalbuterol.tw.

15 proventil.tw.

16 sultanol.tw.

17 ventolin.tw.

18 xopenex.tw.

19 or/9-18

208 and 19

Beta2-adrenoceptor agonists for dysmenorrhoea (Review)

Copyright $(2012$ The Cochrane Collaboration. Published by John Wiley \& Sons, Ltd. 


\section{Appendix 6. EBM Reviews search strategy}

1 dysmenorrh\$.mp. [mp=title, original title, abstract, mesh headings, heading words, keyword]

2 menstru\$ disorder\$.mp. [mp=title, original title, abstract, mesh headings, heading words, keyword]

3 (pelvi\$ adj5 pain).mp. [mp=title, original title, abstract, mesh headings, heading words, keyword]

4 (painful adj5 menstrua\$).mp. [mp=title, original title, abstract, mesh headings, heading words, keyword]

5 (painful adj5 period\$).mp. [mp=title, original title, abstract, mesh headings, heading words, keyword]

6 or/ $1-5$

7 salbutamol.mp. [mp=title, original title, abstract, mesh headings, heading words, keyword]

8 Beta Adrenergic Receptor Stimulating Agent.mp. [mp=title, original title, abstract, mesh headings, heading words, keyword]

9 or $/ 7-8$

106 and 9

11 from 10 keep 1

\section{H I S T O R Y}

\begin{tabular}{l|l|l}
\hline Date & Event & Description \\
\hline 14 April 2008 & Amended & converted to new review format \\
\hline 28 February 2002 & New citation required and major changes & Substantive amendment \\
\hline
\end{tabular}

\section{CONTRIBUTIONSOFAUTHORS}

Zbys Fedorowicz, Vanitha A Jagannath, Akshay Sharma, Jessica H Beaman, Kiran Ejaz and Esther van Zuuren were responsible for:

- organising the retrieval of papers;

- writing to authors of papers for additional information;

- screening search results;

- screening retrieved papers against inclusion criteria;

- appraising the quality of papers;

- data collection for the review;

- extracting data from papers;

- obtaining and screening data on unpublished studies.

ZF, VJ and EvZ entered trial details into RevMan and were responsible for interpretation of the data.

KE participated in study selection and risk of bias assessment.

All review authors contributed to writing the review.

$\mathrm{MN}$ and ZF were responsible for designing and co-ordinating the review and for data management for the review.

$\mathrm{MN}$ and $\mathrm{ZF}$ conceived the idea for the review and are the guarantors for the review.

Beta2-adrenoceptor agonists for dysmenorrhoea (Review) 


\section{DECLARATIONSOF INTEREST}

There are no financial conflicts of interest and the review authors declare that they do not have any associations with any parties who may have vested interests in the results of this review.

\section{DIFFERENCES BETWEEN PROTOCOLANDREVIEW}

The inclusion criteria in the review were amended to include studies where the intervention could have been used as an add-on to standard treatment. This would then allow for the evaluation of studies which could provide evidence of the effects of the intervention in unresponsive dysmenorrhoea.

\section{INDEX TERMS}

\section{Medical Subject Headings (MeSH)}

Acetaminophen [therapeutic use]; Adrenergic beta-2 Receptor Agonists [*therapeutic use]; Caffeine [therapeutic use]; Dysmenorrhea [*drug therapy]; Isoxsuprine [therapeutic use]; Metaproterenol [analogs \& derivatives; therapeutic use]; Randomized Controlled Trials as Topic; Ritodrine [therapeutic use]; Terbutaline [therapeutic use]

\section{MeSH check words}

Adolescent; Adult; Female; Humans; Young Adult 